\title{
3-Aryl Substituted 1-Methylquinolinium Salts as Carbene Precursors
}

\author{
Batsyts, Sviatoslav
}

2019-02-14

Batsyts , S , Vedmid , R , Namyslo, J C , Nieger , M \& Schmidt, A 2019 , ' 3-Aryl

Substituted 1-Methylquinolinium Salts as Carbene Precursors ' , European Journal of Organic Chemistry , no. 6 , pp. 1301-1310 . https://doi.org/10.1002/ejoc.201801648

http://hdl.handle.net/10138/324829

https://doi.org/10.1002/ejoc.201801648

unspecified

acceptedVersion

Downloaded from Helda, University of Helsinki institutional repository.

This is an electronic reprint of the original article.

This reprint may differ from the original in pagination and typographic detail.

Please cite the original version. 


\title{
3-Aryl Substituted 1-Methylquinolinium Salts as Carbene Precursors
}

\author{
Sviatoslav Batsyts, ${ }^{\left[{ }^{[a]}\right.}$ Roman Vedmid, ${ }^{[a]}$ Jan C. Namyslo, ${ }^{[a]}$ Martin Nieger, ${ }^{[b]}$ and Andreas Schmidt*[a]
}

Abstract: 1-Methylquinolinium salts substituted at the C3 position with phenyl, naphthalen-1-yl, phenanthren-9-yl, and pentaphenyl phenyl as well as aryl ethynyl substituents were prepared. Whereas the phenyl, naphthalen-1-yl and phenanthren-9-yl substituents are in conjugation with the quinolinium ring, the sterically crowded pentaphenyl phenyl residue causes a propeller-type molecule possessing a calculated dihedral angle between aryl substituent and the quinolinium ring of approximately $54^{\circ}$. The different influences of the substituents including the aryl ethynyl residues on the $\pi$ architecture is well reflected in their frontier orbital profiles, their spectroscopic properties and in their ability to form $\mathrm{N}$-heterocyclic carbenes. The latter were generated from the C3-aryl substituted quinolinium salts and trapped as sulfur and selenium adducts, whereas the aryl ethynyl substituted derivatives failed to undergo the $\mathrm{NHC}$ generation under the conditions applied. ${ }^{77} \mathrm{Se} \mathrm{NMR}$ measurements of the corresponding selenones reveal that quinolin-2ylidenes are electron poor carbenes with strong $\pi$-acceptor character.

\section{Introduction}

The first stable carbene $\mathbf{A}$ was described by Bertrand et al. in $1988^{[1]}$. Although a strong multiple-bond character of the P-C bond stabilizes $\mathbf{A}$, it can react as a carbene species (Scheme 1). Three years later, Arduengo et al. ${ }^{[2]}$ reported on the N-heterocyclic carbene B which was synthesized by deprotonation of 1,3-di-1adamantylimidazolium chloride with sodium hydride. Meanwhile a great variety of sterically and electronically diverse carbenes have been reported. ${ }^{[3]}$ Nowadays, NHCs are used as starting materials for heterocycle synthesis, ${ }^{[4]}$ as highly efficient ligands for metalcatalyzed reactions ${ }^{[5]}$ and as organocatalysts. ${ }^{[6]}$ Several synthetic procedures to generate NHCs I from cyclic precursors such as heterocumulene adducts II, hetarenium salts III, partially saturated systems IV, mesomeric betaines $\mathbf{V}$, and thiones $\mathbf{V I}$ are known. ${ }^{[7]}$ In comparison to the widely applied and structurally diverse five-membered NHCs, the chemistry of six-membered $\mathrm{NHCs}$ is less explored. Pyridin-2-ylidene 1, proposed as a reactive intermediate of the Hammick reaction ${ }^{[8]}$ as early as 1937 , was later identified by mass spectroscopy combined with computational quantum chemistry ${ }^{[9]}$ (Scheme 2).

[a] S. Batsyts, R. Vedmid, Dr. J. C Namyslo, Prof. Dr. A. Schmidt Clausthal University of Technology, Institute of Organic Chemistry, Leibnizstrasse 6, D-38678 Clausthal-Zellerfeld (Germany)

Fax:(+ 49)-5323-722834

E-mail: schmidt@ioc.tu-clausthal.de

http://www.ioc.tu-clausthal.de

[b] Dr. M. Nieger

University of Helsinki,

Department of Chemistry

P. O. Box 55 (A.I. Virtasen aukio 1), 00014 Helsinki (Finland)

Supporting information for this article is given via a link at the end of the document.
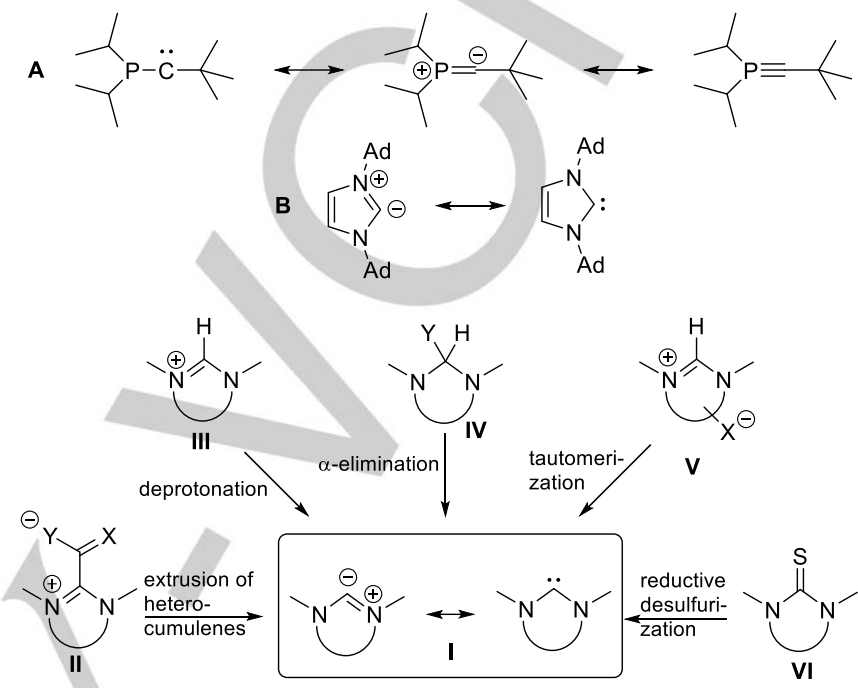

Scheme 1. Preparative routes for the synthesis of NHCs I from cyclic precursors.

Palladium complexes of pyridin-2-ylidene 1 and pyridin-4-ylidene 2 as well as of their related quinolines were prepared from the corresponding chlorohetarenium salts and $\mathrm{Pd}\left(\mathrm{PPh}_{3}\right)_{4}$ by oxidative addition and, furthermore, they were screened for their catalytic activities. ${ }^{[10]}$ Other transition metal complexes such as nickel, ${ }^{[11]}$ osmium, ${ }^{[12]}$ and iridium ${ }^{[13]}$ complexes have been described as well. Several trapping reactions of these carbenes have been performed. ${ }^{[14]}$ Pyridin-4-ylidene 2 can be classified as remote $\mathrm{NHC}$ and can be drawn with an electron sextet structure in analogy to normal NHCs, whereas pyridin-3-ylidene, another remote $\mathrm{NHC}$, can only be presented by dipolar structure 3 . The pyrazin-2-ylidene 4 was formed by decarboxylation of pyrazinium2-carboxylate ${ }^{[15]}$ as precursor which is a pseudo-cross-conjugated mesomeric betaine (PCCMB). Mass spectrometric examinations have been performed. ${ }^{16]}$ Phthalazine can form a carbene complex with palladium by insertion into chlorophthalazine. This enables the formation of phthalazine derivatives of $5 .{ }^{[17,18]}$
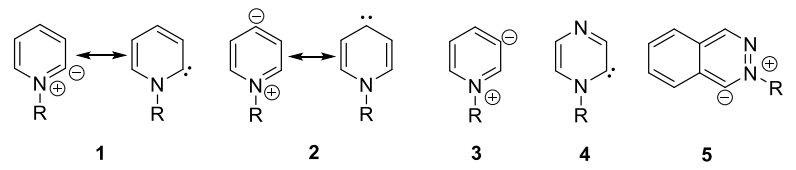

Scheme 2. Six-membered N-heterocyclic carbenes.

The pyridinium salt precursors of the NHCs $\mathbf{1}, \mathbf{2}$, and $\mathbf{3}$ possess a reduced electron density at position $2 / 6$ and 4 as evidenced by the mesomeric structures of $\mathrm{N}$-methylpyridinium (Scheme 3 ). The total electron deficiency was calculated to be +0.165 (C4) and 
$+0.241(\mathrm{C} 2 / 6)$, respectively. ${ }^{[19]}$ The lowest unoccupied molecular orbital (LUMO) resembles $\psi_{4}{ }^{*}$ of benzene. Its polarization, however, causes a considerably larger coefficient at C4 than at C2/6. ${ }^{[20]}$ Quinolinium salts show similar properties.

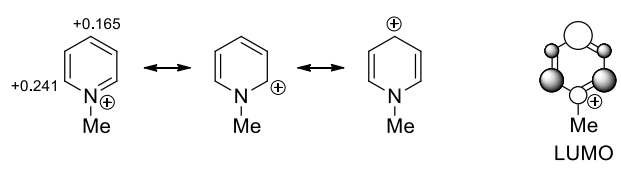

Scheme 3. Total electron deficiency, resonance structures, and the LUMO of $\mathrm{N}$-methylpyridinium.

To the best of our knowledge stable quinolinium carbenes are not known, and only a few carbene complexes have been synthesized so far. ${ }^{[10,21]}$ Some examples are given in Scheme 4.

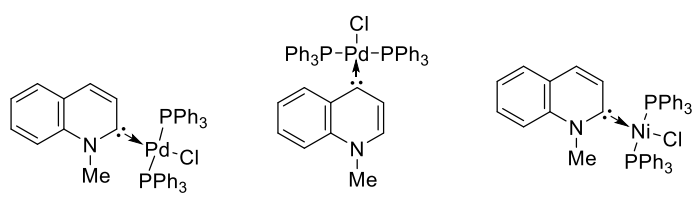

Scheme 4. Quinoline carbene complexes.

In continuation of our interest in the different types of conjugation and their consequences for $\mathrm{N}$-heterocyclic carbene formation ${ }^{[7,22]}$ we examined the influence of substituents at $\mathrm{C} 3$ of quinolinium on trapping reactions of quinolin-2-ylidenes generated from them. We chose different aryl and ethynyl substituents which are in conjugation to the quinolinium ring. These are, in summary, slight electron donators in terms of Hammett's substituent constants (Ph: $\left.\sigma_{\mathrm{m}}+0.06, \sigma_{\mathrm{p}}-0.01 ;-\mathrm{C} \equiv \mathrm{C}-\mathrm{Ph}: \sigma_{\mathrm{m}}+0.14, \sigma_{\mathrm{p}}+0.16\right) .{ }^{\left[{ }^{[3]} \text { However, }\right.}$ they cause different $\pi$-architectures of the quinolinium salts which take influence of the spectroscopic properties as well as on the chemistry of the compounds in terms of $\mathrm{NHC}$ generation.

\section{Results and Discussion}

\section{Syntheses of 3-substituted quinolinium salts}

To prepare the starting compounds, 3-bromoquinoline 6 was subjected to a Suzuki-Miyaura reaction with aryl boronic acids in the presence of $10 \% \mathrm{Pd}\left(\mathrm{CH}_{3} \mathrm{COO}\right)_{2}$ as a catalyst and $\mathrm{K}_{3} \mathrm{PO}_{4}$ as a base in toluene/water solution to give 3-aryl quinolines 8a-c (Scheme 5). Related to previous works, ${ }^{[22 c, 24]}$ dimethyl sulfate was used as a convenient reagent for the quaternization of $8 \mathbf{a}-\mathbf{c}$ to give the salts $9 \mathbf{a}-\mathbf{c}$ in high yields. $50 \mu \mathrm{l}$ of nitrobenzene were used as a catalyst in these reactions. The syntheses of the 3-alkynyl substituted quinolinium salts 9 d-f were accomplished by Sonogashira coupling reactions with the corresponding acetylenes 7 and 3-bromoquinoline 6 , followed by methylation. Compound $\mathbf{8 d}$ was converted into $\mathbf{8} \mathbf{g}$ by a Diels-Alder reaction with pentaphenylcyclopentenone. Methylation employing the aforementioned method gave the quinolinium derivative $\mathbf{9 g}$ in almost quantitative yields. Due to mutual repulsion and steric hindrance of the side wings the molecule has to adopt a propellertype conformation.

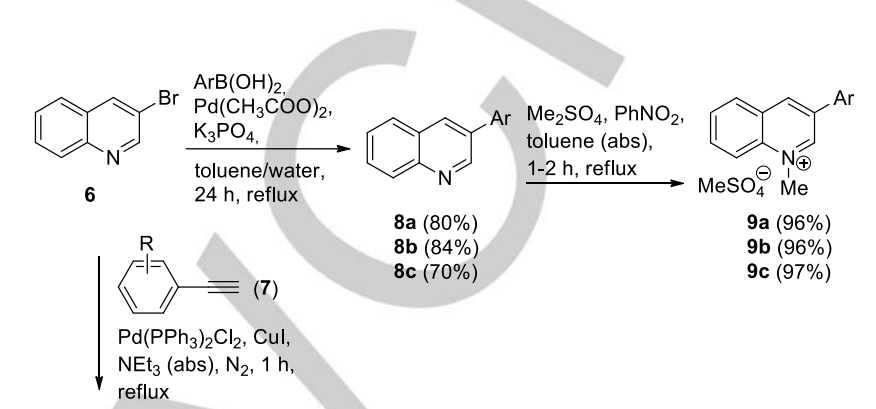

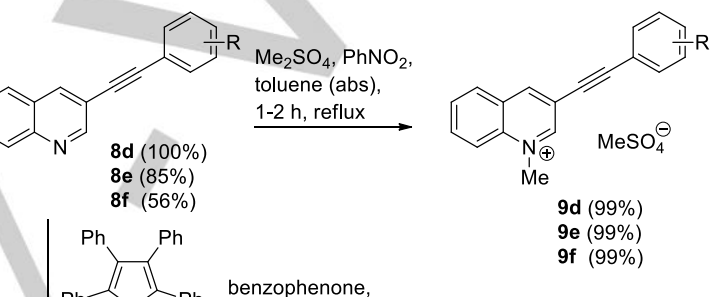

[8d]

Penzophenone

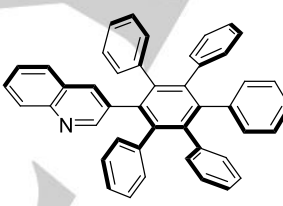

$8 \mathrm{~g}(51 \%)$

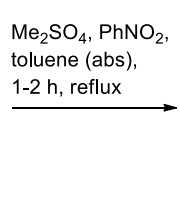

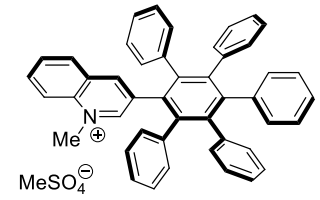

9g $(99 \%)$

$\mathrm{Ar}=$ phenyl (a), naphthalen-1-yl (b), phenanthren-9-yl (c) $\mathrm{R}=\mathrm{H}(\mathbf{d}), p$-COOMe (e), o-COOMe (f).

Scheme 5. Synthesis of 3 -substituted quinolines $8 \mathbf{a}-\mathbf{f}$ by cross-coupling reactions and 1-methyl-3-substituted quinolinium methylsulfates $\mathbf{9 a - g}$.

Different ${ }^{13} \mathrm{C}$ NMR resonance frequencies of $\mathrm{C} 3$ of the quinolinium ring are observed. Whereas the 3-aryl substituted quinolinium salts $9 \mathrm{a}-\mathrm{c}$ and $\mathbf{9 g}$ show chemical shifts of their $\mathrm{C} 3$ carbon atom between $133.4 \mathrm{ppm}$ and $134.4 \mathrm{ppm}$, the 3-ethynyl substituted compounds show signals between 116.2 and $116.8 \mathrm{ppm}$. Correspondingly, the natural charges at C3 were calculated to be $-0.067(9 a),-0.129(9 d)$ and -0.063 (9g) [B3LYP 6-31G(d,p)]. All other data of calculated NMR shifts as well as natural charges of the model compounds $\mathbf{9 a}, \mathbf{9} \mathbf{d}$ and $\mathbf{9 g}$ are presented in the supplementary material. We also calculated the most stable conformations and frontier orbitals. The dihedral angle between benzene and quinoline rings in salt $9 \mathrm{a}$ was calculated to be $37.9^{\circ}$ in vacuo. The corresponding angle in the propeller-type salt $9 \mathrm{~g}$ is larger and has a value of $54.1^{\circ}$. The five other phenyl rings are twisted from $66^{\circ}$ to $68^{\circ}$ with respect to the central phenyl ring. In the ethynyl substituted salt $\mathbf{9 d}$ the two aromatics are planar with respect to each other. The calculated $\mathrm{C}_{3}-\mathrm{C}_{\mathrm{Ar}}$ bond lengths in $9 \mathrm{a}$ 
and $\mathbf{9 g}$ is comparable and adopt values of $148.0 \mathrm{pm}$ and 149.4 $\mathrm{pm}$, respectively. The calculated $\mathrm{C}_{3}-\mathrm{C}_{\mathrm{sp}} \alpha$ bond length of $9 \mathrm{~d}$ is shorter with a value of $141.2 \mathrm{pm}$. The HOMO/LUMO profiles are shown in Figure 1. The coefficients of the lowest unoccupied molecular orbital (LUMO) of $\mathbf{9 a}$ and $\mathbf{9 g}$ are exclusively located in the quinolinium ring. In salt $\mathbf{9 d}$ a small LUMO coefficient is also located on the $\mathrm{C} \beta$ carbon atom of the $\mathrm{C} \equiv \mathrm{C}$ triple bond. The $\mathrm{HOMO}$ of the propeller-type salt $\mathbf{9 g}$ is strictly separated from the LUMO's coefficients and is located on the opposite side of the molecule. Coefficients of the HOMOs of $9 a$ and $9 d$ are distributed in the entire molecules with their highest coefficients in the substituents at C3. The calculated band gaps have values of 3.36, 2.24, and $2.67 \mathrm{eV}$ for $9 \mathbf{a}, \mathbf{9 g}$, and $\mathbf{9 d}$, respectively.

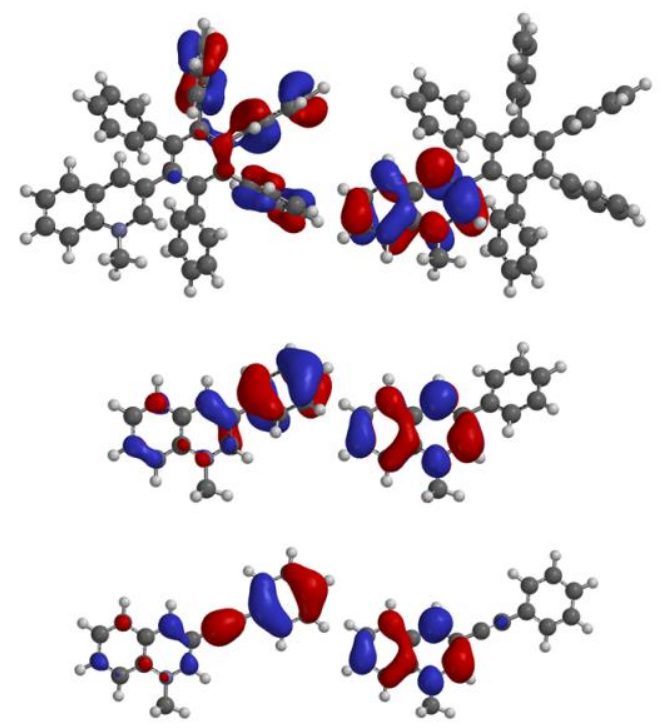

Figure 1. HOMO (left) and LUMO (right) profiles of carbene precursors $\mathbf{9 g}$ (above), 9a (middle), and 9d (below).

\section{NHC formations and trapping reactions}

First, deprotonations of the quinolinium salts were examined by base screenings in combination with NMR experiments. To increase the solubility of the salt $9 c$, which is insoluble in toluene or benzene, the methylsulfate anion was exchanged to hexafluorophosphate by precipitation in aqueous solution. Addition of potassium tert-pentoxide or LiHMDS to a solution of salt $9 \mathrm{c}$ in $\left[\mathrm{D}_{6}\right] \mathrm{DMSO},\left[\mathrm{D}_{8}\right] \mathrm{THF}$ or $\left[\mathrm{D}_{3}\right] \mathrm{MeCN}$ at room temperature led to an upfield shift of the resonance frequencies, however, considerable amounts of decomposition products were visible in the spectra. By contrast, the base LiHMDS in $\left[D_{8}\right]$ THF formed the adduct $\mathbf{1 3}$ of the electrophilic iminium moiety of $9 \mathrm{CPF}_{6}$ (Scheme $6)$.

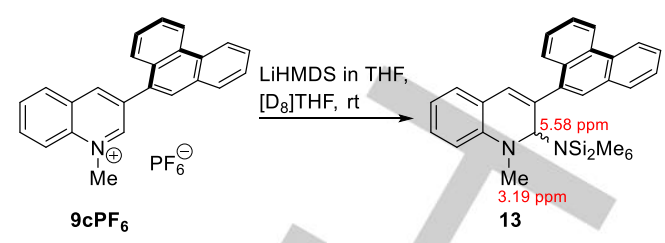

Scheme 6. The reaction of salt $9 \mathrm{cPF}_{6}$ with LiHMDS in $\left[\mathrm{D}_{8}\right] \mathrm{THF}$ and characteristic chemical shifts.

Next we performed a series of reactions to deprotonate the quinolinium salt $9 \mathrm{c}$ at $\mathrm{C} 2$ in the presence of elemental sulfur and to form thiones as trapping products of the corresponding $\mathrm{N}$ heterocyclic carbenes. Lithium hydroxide gave no reaction (Scheme 7, A). Potassium tert-butoxide ${ }^{[25]}$ in anhydrous toluene under reflux conditions gave the quinolinone 10, presumably formed by addition of the base at $\mathrm{C} 2$ followed by thermal elimination of isobutene. The $\mathrm{C} 2$ carbon atom of $\mathbf{1 0}$ gives the signal at $162.0 \mathrm{ppm}$ in the ${ }^{13} \mathrm{C}$ NMR spectrum. Interaction of $9 \mathrm{c}$ and potassium tert-pentoxide in acetonitrile has been examined by HRMS. The electrospray ionization mass shows the molecular peak at $m / z=374.0948$, which corresponds to the potassium adduct $\mathbf{1 0} \cdot \mathrm{K}^{+}$. The addition of $\mathrm{LiOH}$ did not improve the yield (Scheme 7, B and C). However, using potassium tert-pentoxide led to a color change during the reaction and gave thione 11c as an orange solid in $20 \%$ yield. Addition of 1.2 equiv of potassium tert-pentoxide and 10 equiv of $\mathrm{LiOH}$ increased the yield to $90 \%$. Exchanging $\mathrm{LiOH}$ to $\mathrm{LiBr}$ also led to an increased yield 11c compared to conditions without lithium compounds. However, potassium tert-pentoxide proved vital as $\mathrm{LiOH}$ alone did not give successful results as described above (Scheme 7, D-F).

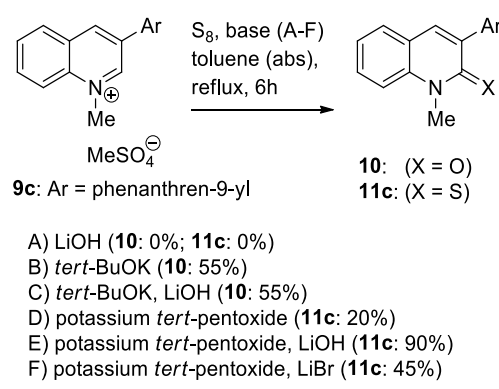

Scheme 7. Trapping reaction of salt $9 \mathrm{c}$ with sulfur under different conditions.

Applying the optimized reaction condition to $9 a, b, g$ gave the corresponding thiones $\mathbf{1 1 a , b , g}$ as orange colored solids, however, in lower yields (Scheme 8). The same conditions for the generation of $\mathrm{NHCs}$ and their trapping with sulfur have been applied to the 3-ethynyl substituted quinolinium salts $\mathbf{9 d - f}$, however, without results. No reaction occurred. 


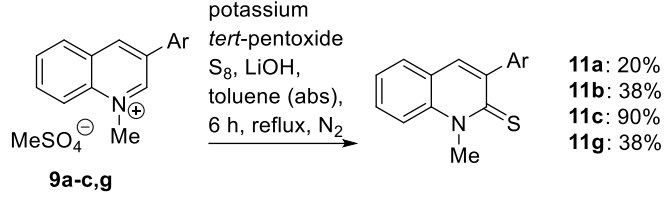

$\operatorname{Ar}=$ phenyl (a), naphthalen-1-yl (b), phenanthren-9-yl (c), pentaphenylbenzene $(d)$

Scheme 8. Trapping reactions of NHCs generated from the salts $9 \mathbf{a}-\mathbf{c}$ and $\mathbf{9 g}$ with sulfur by optimized conditions.

The resonance frequencies of the $\mathrm{C}=\mathrm{S}$ carbon atoms in $\mathrm{CDCl}_{3}$ are shifted downfield compared to the $\mathrm{C}=\mathrm{O}$ signal of $\mathbf{1 0}$ and adopt values of 185.0, 185.4, and $185.3 \mathrm{ppm}$, respectively. The structure of 11b was additionally proven by X-ray structure analysis (Figure 2). Single crystals of thione 11b were obtained by slow evaporation of a concentrated solution in chloroform. The compound crystallized orthorhombic. The CS bond has a length of $168.39(18) \mathrm{pm}$. The typical $\mathrm{C}\left(\mathrm{sp}^{2}\right)=\mathrm{S}$ double bond length is in the range from 159.9 to $161.1 \mathrm{pm}$, whereas the $\mathrm{C}\left(\mathrm{sp}^{2}\right)-\mathrm{S}$ single bond length is $177 \mathrm{pm} .{ }^{[26]}$ The dihedral angle between the quinoline and naphthalene planes is $77.56(3)^{\circ}$.

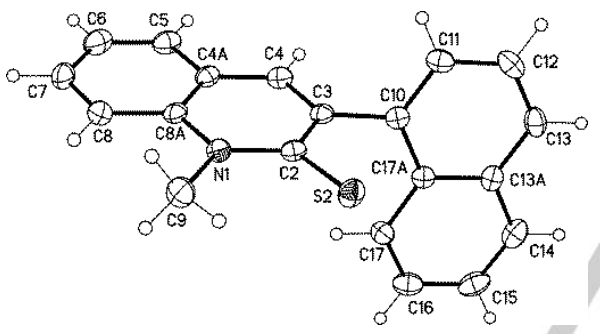

Figure 2. Molecular structure of thione 11b (displacement parameters are drawn at $50 \%$ probability level).

Reactions of the 3-substituted quinolinium salts 9a-c with elemental selenium in the presence of potassium tert-pentoxide gave the selenones 12a-c (Scheme 9). All selenones are red compounds with a specific odor. Furthermore, the bulkier the aromatic substituent, the higher the melting point in thiones and selenones. Thus, the selenone with a phenanthrenyl residue (12c) has a melting point of $271^{\circ} \mathrm{C}$, while the selenone with a phenyl residue (12a) is an oil.

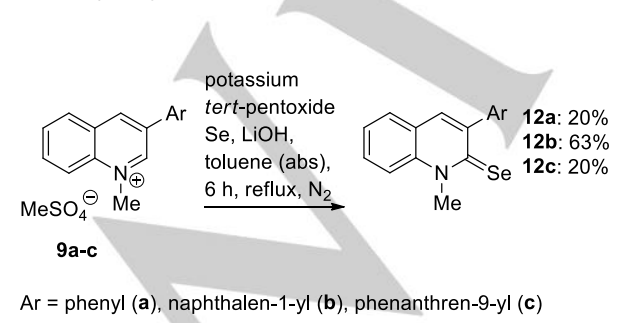

Scheme 9. Trapping reactions of salts 9a-c with selenium.
Whereas the ${ }^{13} \mathrm{C}$ chemical shifts of the $\mathrm{C} 2$ atoms of the selenones 12a-c are very close to the $\mathrm{C} 2$ carbon atoms of the thiones 11ac, the resonance frequencies of methyl groups differ significantly (c.f. supplementary material). The structure of $12 \mathrm{c}$ was proven by X-ray structure analysis (Figure 3 ). Single crystals of selenone 12c were obtained by diffusion of cyclohexane into its solution in chloroform. The compound crystallized monoclinic. The $\mathrm{C}=\mathrm{Se}$ bond has a length of $183.8(2) \mathrm{pm}$. The typical $\mathrm{C}\left(\mathrm{sp}^{2}\right)=\mathrm{Se}$ double bond length is $171 \mathrm{pm}^{[27]}$ and the typical $\mathrm{C}\left(\mathrm{sp}^{2}\right)$-Se single bond length is $189 \mathrm{pm}^{[26]}$. The dihedral angle between the quinoline and naphthalene planes is $70.08(3)^{\circ}$.

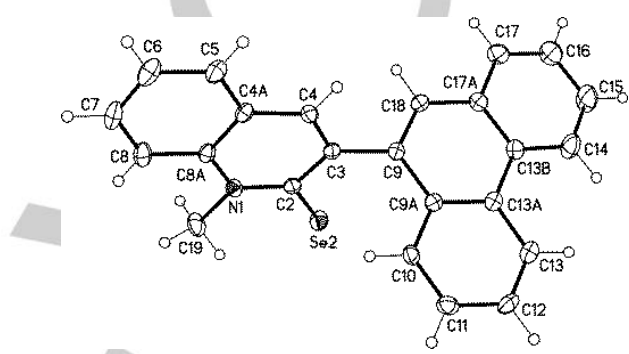

Figure 3. Molecular structure of selenone 12c (displacement parameters are drawn at $50 \%$ probability level).

Quinoline selenones are rare and little information is available. Only a few compounds have been described so far. ${ }^{[28]}$ The ${ }^{77} \mathrm{Se}$ NMR spectra for 12a-c were measured and the signals of Se atoms were found at $706.6,716.8$, and 720.4 ppm, respectively. These downfield shifts indicate electron poor former carbenes with strong $\pi$-acceptor character (Scheme 10). ${ }^{[29]}$ The ${ }^{77} \mathrm{Se}$ chemical shift of 1-methylquinoline-2(1H)-selenone has a value of 646.7 ppm. ${ }^{[28 \mathrm{c}]}$

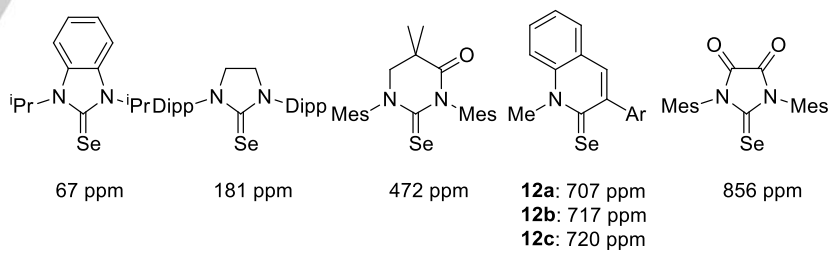

Scheme 10. Comparison of Ganter's NHC selenium adducts. ${ }^{77}$ Se chemical shifts of $12 a-c$.

\section{Conclusions}

Aryl as well as ethynyl substituents at position 3 of quinolinium salts were prepared. Whereas the former mentioned underwent trapping reactions of their $\mathrm{N}$-heterocyclic carbenes with sulfur and selenium, respectively, the latter failed to undergo these reactions. This work supplements results concerning the electrophilicities vs. acidities in six-membered $\mathrm{N}$-heterocyclic carbene precursors. ${ }^{[30]}$ 


\section{Experimental Section}

All reactions were carried out under an atmosphere of nitrogen in flame or oven-dried glassware. All chemicals were purchased and used without further purification unless otherwise mentioned. Anhydrous solvents were dried according to standard procedures before usage. Melting points are uncorrected and were determined in an apparatus according to Dr. Tottoli (Büchi). The ATR-IR spectra were obtained on a Bruker Alpha in the range of 400 to $4000 \mathrm{~cm}^{-1}$. ${ }^{1} \mathrm{H}$ NMR spectra were recorded at $400 \mathrm{MHz}$ or 600 $\mathrm{MHz} .{ }^{13} \mathrm{C}$ NMR spectra were recorded at $100 \mathrm{MHz}$ or $150 \mathrm{MHz}$, with the solvent peak used as the internal reference. Multiplicities are described by using the following abbreviations: $\mathrm{s}=$ singlet, $\mathrm{d}=$ doublet, $\mathrm{t}=$ triplet, $\mathrm{q}=$ quartet, and $\mathrm{m}=$ multiplet. Signal orientations in DEPT experiments were described as follows: $0=$ no signal; + = up $\left(\mathrm{CH}, \mathrm{CH}_{3}\right) ;-=$ down $\left(\mathrm{CH}_{2}\right) .{ }^{77} \mathrm{Se}$ NMR spectra were recorded at $114 \mathrm{MHz}$. The electrospray ionization mass spectra (ESIMS) were measured with a Varian 320 MS Triple Quad GC/MS/MS (EIMS) or with an Agilent LCMSD series HP 1100 with APIES at fragmentor voltages as indicated. Samples were sprayed from $\mathrm{MeOH}$ at $4000 \mathrm{~V}$ capillary voltage and fragmentor voltages of $30 \mathrm{~V}$ unless otherwise noted. The HR-MS spectra were obtained with a Bruker Impact II, a Bruker Daltonik Tesla-Fourier transform-ion cyclotron resonance mass spectrometer, or with a Waters Micromass LCT with the direct inlet. Chromatography: The reactions were traced by thin layer chromatography with silica gel 60 (F254, company MERCK KGAA). For the detection of substances, quenching was used at either $254 \mathrm{~nm}$ or $366 \mathrm{~nm}$ with a mercury lamp. The preparative column chromatography was conducted through silica gel 60 (230 400 mesh) of the company MERCK KGAA.

\section{Crystal Structure Determinations of $11 b$ and $12 c$}

The single-crystal X-ray diffraction studies were carried out on a Bruker D8 Venture diffractometer with Photon100 detector at 123(2) K using Cu-Ka radiation $\left(\lambda=1.54178 \AA\right.$. Direct Methods (SHELXS-97) ${ }^{[31]}$ were used for structure solution and refinement was carried out using SHELXL-2014 (full-matrix least-squares on $F^{2}$ ) ${ }^{[32]}$. Hydrogen atoms were localized by difference electron density determination and refined using a riding model. Semi-empirical absorption corrections were applied. 11c was refined as an inversion twin (see cif-file for details).

11b: yellow crystals, $\mathrm{C}_{20} \mathrm{H}_{15} \mathrm{NS}, M_{\mathrm{r}}=301.39$, crystal size $0.20 \times 0.18 \times$ $0.10 \mathrm{~mm}$, orthorhombic, space group $P 2{ }_{1} 2{ }_{1} 2_{1}$ (no. 19), $a=8.3590$ (5) $\AA$, $b$ $=12.9270(7) \AA, c=13.7331(7) \AA, V=1483.95(14) \AA^{3}, Z=4, \rho=1.349$ $\mathrm{Mg} / \mathrm{m}^{-3}, \mu\left(\mathrm{Cu}-\mathrm{K}_{\mathrm{a}}\right)=1.874 \mathrm{~mm}^{-1}, F(000)=632,2 \theta_{\max }=144.2^{\circ}, 10197$ reflections, of which 2906 were independent $\left(R_{\text {int }}=0.021\right), 201$ parameters $R_{1}=0.026$ (for $2872 \mathrm{I}>2 \sigma(\mathrm{I})$ ), $\mathrm{w} R_{2}=0.072$ (all data), $S=1.05$, largest diff.

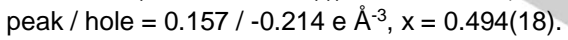

12c: red crystals, $\mathrm{C}_{24} \mathrm{H}_{17} \mathrm{NSe}, M_{\mathrm{r}}=398.34$, crystal size $0.22 \times 0.06 \times 0.02$ $\mathrm{mm}$, monoclinic, space group $P 2_{1} / \mathrm{c}$ (no. 14), $a=6.1638(2) \AA, b=$ 19.9050(6) $\AA, c=14.3978(4) \AA, \beta=95.257(1)^{\circ}, V=1759.04(9) \AA^{3}, Z=4$, $\rho=1.504 \mathrm{Mg} / \mathrm{m}^{-3}, \mu\left(\mathrm{Cu}-\mathrm{K}_{\alpha}\right)=2.920 \mathrm{~mm}^{-1}, F(000)=808,2 \theta_{\max }=144.2^{\circ}$, 16462 reflections, of which 3473 were independent $\left(R_{\mathrm{int}}=0.033\right), 236$ parameters, $R_{1}=0.031$ (for $3138 \mathrm{I}>2 \sigma(\mathrm{I})$ ), $w R_{2}=0.082$ (all data), $S=$ 1.05, largest diff. peak / hole $=0.437 /-0.366$ e $\AA^{-3}$.

CCDC 1874495 (11b), and 1874496 (12c) contain the supplementary crystallographic data for this paper. These data can be obtained free of charge from The Cambridge Crystallographic Data Centre via www.ccdc.cam.ac.uk/data_request/cif.

Calculations: All density-functional theory (DFT)-calculations were carried out by using the Spartan $\AA$ Software (Spartan'16, Wavefunction, Inc., Irvine, CA. Available from: http://www.wavefun.com) running on an
Intel ${ }^{\circledR}$ Core $^{\mathrm{TM}}$ i7-6950X deca core system equipped with 64 GB RAM main memory and sufficient solid-state disc space. MMFF optimized structures were used as starting geometries. Then, the B3LYP density functional and the $6-31 \mathrm{G}(\mathrm{d}, \mathrm{p})$ basis set was used in order to allow for comparison with previous results. All final structures were proven to be true minima by the absence of imaginary frequencies or transition states by the occurrence of a negative frequency.

General procedure for the preparation of the 3-arylquinolines (Procedure 1; Suzuki-Miyaura coupling): Under a nitrogen atmosphere, $1.040 \mathrm{~g}(5.00 \mathrm{mmol})$ of 3 -bromoquinoline 6 were dissolved in $20 \mathrm{~mL}$ of anhydrous toluene unless otherwise noted and treated with $10-\mathrm{mol}-\%$ of $\mathrm{Pd}\left(\mathrm{CH}_{3} \mathrm{COO}\right)_{2}$ The mixtures were then subjected to ultrasound irradiation for $5 \mathrm{~min}$ and then stirred at $\mathrm{rt}$ for additional $0.5 \mathrm{~h}$. Then $6.00 \mathrm{mmol}$ of the boronic acid, $40.00 \mathrm{mmol}$ of potassium phosphate and $4 \mathrm{~mL}$ of water were added, and the mixtures were heated under reflux for $24 \mathrm{~h}$. The mixtures were then allowed to cool to $\mathrm{rt}$, treated with dichloromethane $(50 \mathrm{~mL})$ and washed with water. The organic phases were then dried over $\mathrm{MgSO}_{4}$ and filtered, and the solvents were removed in vacuo. The resulting residues were finally purified by column chromatography (petroleum ether: ethyl acetate) to afford the products.

3-Phenylquinoline (8a): According to Procedure 1, a solution of $1.040 \mathrm{~g}$ $(5.00 \mathrm{mmol})$ of 3-bromoquinoline $6,0.112 \mathrm{~g}(0.50 \mathrm{mmol})$ of $\mathrm{Pd}\left(\mathrm{CH}_{3} \mathrm{COO}\right)_{2}$, $0.732 \mathrm{~g}(6.00 \mathrm{mmol})$ phenylboronic acid and $8.480 \mathrm{~g}(40.00 \mathrm{mmol})$ of $\mathrm{K}_{3} \mathrm{PO}_{4}$ in $54 \mathrm{~mL}$ of the toluene-water mixture was heated for $24 \mathrm{~h}$ under reflux temperature. Finally, a purification by column chromatography (petroleum ether: ethyl acetate $=3: 1$ ) gave 3-phenylquinoline $8 \mathbf{a}$. Yield $0.820 \mathrm{~g}, 80 \%$, a brownish solid, m.p. $50-51^{\circ} \mathrm{C} .{ }^{1} \mathrm{H} \mathrm{NMR}\left(600 \mathrm{MHz}, \mathrm{CDCl}_{3}\right)$ : $\delta=9.20(\mathrm{~s}, 1 \mathrm{H}, 2-\mathrm{H}), 8.31(\mathrm{~d}, J=1.8 \mathrm{~Hz}, 1 \mathrm{H}, 4-\mathrm{H}), 8.16(\mathrm{~d}, J=8.3 \mathrm{~Hz}, 1 \mathrm{H}$, $8-\mathrm{H}), 7.89$ ( d, J = 8.1 Hz, 1H, 5-H), 7.74-7.71 (m, 3H, 7-H, 2'-H, 6'-H), 7.58 (t, $J=7.4 \mathrm{~Hz}, 1 \mathrm{H}, 6-\mathrm{H}), 7.54-7.52\left(\mathrm{~m}, 2 \mathrm{H}, 3^{\prime}-\mathrm{H}, 5^{\prime}-\mathrm{H}\right), 7.44(\mathrm{~m}, 1 \mathrm{H}$, $\left.4^{\prime}-\mathrm{H}\right)$ ppm. ${ }^{13} \mathrm{C}$ NMR (150 MHz, $\left.\mathrm{CDCl}_{3}\right): \delta=150.0(+, \mathrm{C} 2), 147.4$ (o, C8a), 138.0 (o, C1'), 134.0 (o, C3), 133.4 (+, C4), 129.5 (+, C7), 129.3 (o, C4a), $129.3\left(+, \mathrm{C} 8, \mathrm{C}^{\prime}, \mathrm{C}^{\prime}\right), 128.3\left(+, \mathrm{C}^{\prime}\right), 128.2(+, \mathrm{C} 5), 127.6\left(+, \mathrm{C}^{\prime}, \mathrm{C}^{\prime}\right)$, 127.2 (+, C6) ppm. IR (ATR): 3027, 1568, 1492, 1368, 1340, 1027, 952 , $914,861,785,773,762,693,624,612,560,545,494,479,443 \mathrm{~cm}^{-1}$. Spectroscopic data are in agreement with those reported in the literature. ${ }^{[33]}$

3-(Naphthalen-1-yl)quinoline (8b): According to Procedure 1, a solution of $1.040 \mathrm{~g}(5.00 \mathrm{mmol})$ of 3-bromoquinoline $6,0.112 \mathrm{~g}(0.50 \mathrm{mmol})$ of $\mathrm{Pd}\left(\mathrm{CH}_{3} \mathrm{COO}\right)_{2}, 1.032 \mathrm{~g}(6.00 \mathrm{mmol})$ naphthalene-1-boronic acid and 8.480 $\mathrm{g}(40.00 \mathrm{mmol})$ of $\mathrm{K}_{3} \mathrm{PO}_{4}$ in $54 \mathrm{~mL}$ of the toluene-water mixture was heated for $24 \mathrm{~h}$ under reflux temperature. Finally, a purification by column chromatography (petroleum ether: ethyl acetate $=3: 1$ ) gave 3(naphthalen-1-yl)quinoline $8 \mathrm{~b}$. Yield $1.071 \mathrm{~g}, 84 \%$, a white solid, m.p. 57$58{ }^{\circ} \mathrm{C} .{ }^{1} \mathrm{H}$ NMR $\left(600 \mathrm{MHz}, \mathrm{CDCl}_{3}\right): \delta=9.08(\mathrm{~d}, J=1.9 \mathrm{~Hz}, 1 \mathrm{H}, 2-\mathrm{H}), 8.28$ (d, $J=1.9 \mathrm{~Hz}, 1 \mathrm{H}, 4-\mathrm{H}), 8.23(\mathrm{~d}, J=8.5 \mathrm{~Hz}, 1 \mathrm{H}, 8-\mathrm{H}), 7.97\left(\mathrm{~m}, 1 \mathrm{H}, 5^{\prime}-\mathrm{H}\right)$, $7.95\left(\mathrm{~m}, 1 \mathrm{H}, 4^{\prime}-\mathrm{H}\right), 7.90(\mathrm{~d}, J=7.9 \mathrm{~Hz}, 1 \mathrm{H}, 5-\mathrm{H}), 7.87(\mathrm{~d}, J=8.5 \mathrm{~Hz}, 1 \mathrm{H}$, 8'-H) 7.79 (ddd, $J=1.4,7.0,8.4 \mathrm{~Hz}, 1 \mathrm{H}, 7-\mathrm{H}$ ), 7.63 (ddd, $J=1.0,7.0,8.0$ $\mathrm{Hz}, 1 \mathrm{H}, 6-\mathrm{H}$ ), 7.60 (dd, $J=7.0,8.2 \mathrm{~Hz}, 1 \mathrm{H}, 3{ }^{\prime}-\mathrm{H}$ ), 7.54 (ddd, $J=1.2,6.7$, $8.0 \mathrm{~Hz}, 1 \mathrm{H}, 6^{\prime}-\mathrm{H}$ ), 7.53 (dd, $J=1.2,7.0 \mathrm{~Hz}, 1 \mathrm{H}, 2^{\prime}-\mathrm{H}$ ), 7.47 (ddd, $J=1.4$, 6.9, 8.4 Hz, $\left.1 \mathrm{H}, 7^{\prime}-\mathrm{H}\right)$ ppm. ${ }^{13} \mathrm{C} \mathrm{NMR}\left(150 \mathrm{MHz} \mathrm{CDCl}_{3}\right): \delta=152.0(+, \mathrm{C} 2)$, 147.4 (о. C8a), 136.5 (+, C4), 136.4 (o, C1'), 134.0 (o, C4a'), 133.9 (o, C3), 131.8 (o, C8a'), $129.8(+, \mathrm{C} 7), 129.4(+, \mathrm{C} 8), 128.8(+, \mathrm{C} 4), 128.7\left(+, \mathrm{C}^{\prime}\right)$, 128.1 (+, C5), 128.0 (o, C4a), $127.9\left(+, \mathrm{C}^{\prime}\right), 127.2(+, \mathrm{C} 6), 126.8\left(+, \mathrm{C}^{\prime}\right)$, $126.3\left(+, C^{\prime}\right), 125.6\left(+, 3^{\prime}\right), 125.5\left(+, C^{\prime}\right)$ ppm. IR (ATR): 3034, 1811, 1566, 1508, 1490, 1396, 1364, 1332, 1125, 1017, 941, 904, 861, 774, 745, $664,616,566,479,450,430 \mathrm{~cm}^{-1}$. Spectroscopic data are in agreement with those reported in the literature. ${ }^{[34]}$

3-(Phenanthren-9-yl)quinoline (8c): According to Procedure 1, a solution of $1.040 \mathrm{~g}(5.00 \mathrm{mmol})$ of 3-bromoquinoline $6,0.112 \mathrm{~g}(0.50 \mathrm{mmol})$ of 
$\mathrm{Pd}\left(\mathrm{CH}_{3} \mathrm{COO}\right)_{2}, 1.332 \mathrm{~g}(6.00 \mathrm{mmol})$ phenanthrene-9-boronic acid and $8.480 \mathrm{~g}(40.00 \mathrm{mmol})$ of $\mathrm{K}_{3} \mathrm{PO}_{4}$ in $54 \mathrm{~mL}$ of the toluene-water mixture was heated for $24 \mathrm{~h}$ under reflux temperature. Finally, a purification by column chromatography (petroleum ether: ethyl acetate $=3: 1$ ) gave 3 (phenanthren-9-yl)quinoline 8c. Yield $1.068 \mathrm{~g}, 70 \%$, a light-yellow oil. ${ }^{1} \mathrm{H}$ $\operatorname{NMR}\left(400 \mathrm{MHz}, \mathrm{CDCl}_{3}\right): \delta=9.12-9.11(\mathrm{~m}, 1 \mathrm{H}), 8.82-8.80(\mathrm{~m}, 1 \mathrm{H}), 8.76-$ $8.74(\mathrm{~m}, 1 \mathrm{H}), 8.32-8.31(\mathrm{~m}, 1 \mathrm{H}), 8.24-8.23(\mathrm{~m}, 1 \mathrm{H})$, 7.94-7.89 $(\mathrm{m}, 2 \mathrm{H})$, 7.88-7.86 (m, $1 \mathrm{H})$, 7.81-7.79 $(\mathrm{m}, 1 \mathrm{H}), 7.78-7.77(\mathrm{~m}, 1 \mathrm{H}), 7.72-7.69(\mathrm{~m}, 2 \mathrm{H})$ 7.66-7.61 (m, 2H), 7.57-7.54 (m, 1H) ppm. ${ }^{13} \mathrm{C} \mathrm{NMR}\left(100 \mathrm{MHz}, \mathrm{CDCl}_{3}\right): \delta$ $=152.1,147.6,136.4,135.1,133.9,132.4,131.0,130.9,130.4,129.8$, $129.5,128.9,128.9,128.1,128.0,127.3,127.2,127.2,127.0,127.0,126.5$, 123.3, 122.8 ppm. IR (ATR): $3058,1732,1618,1566,1489,1449,1337$, $1239,1123,1044,914,891,861,787,743,723,616,537,511,479,427$, $411 \mathrm{~cm}^{-1}$. Spectroscopic data are in agreement with those reported in the literature. ${ }^{[35]}$

\section{General procedure of the Sonogashira-Hagihara couplings (Procedure 2)}

The reactions were carried out under a nitrogen atmosphere. A mixture of $5 \mathrm{mmol}$ of 3-bromoquinoline $6,1 \mathrm{~mol} \%$ of $\mathrm{Pd}\left(\mathrm{PPh}_{3}\right)_{2} \mathrm{Cl}_{2}$, and $2 \mathrm{~mol} \%$ of Cul was suspended in $7 \mathrm{~mL}$ of dry $\mathrm{NEt}_{3}$ with stirring. A sample of the corresponding ethyne 7 (1.05 equiv) in dry $\mathrm{NEt}_{3}$ was added dropwise at ambient temperature. The resulting solutions were then stirred at reflux temperature until complete conversion was monitored by TLC. The mixtures were then allowed to cool to rt. The solvents were removed in vacuo. The resulting residues were finally purified by column chromatography (petroleum ether: ethyl acetate) to afford the products.

3-(Phenylethynyl)quinoline (8d): According to Procedure 2 , a solution of $2.080 \mathrm{~g}(10.00 \mathrm{mmol})$ of 3-bromoquinoline $6,0.070 \mathrm{~g}(0.10 \mathrm{mmol})$ of $\mathrm{Pd}\left(\mathrm{PPh}_{3}\right)_{2} \mathrm{Cl}_{2}, 0.038 \mathrm{~g}(0.20 \mathrm{mmol}) \mathrm{Cul}$ and $1.071 \mathrm{~g}(10.50 \mathrm{mmol})$ of phenylacetylene in $30 \mathrm{~mL}$ of anhydrous $\mathrm{NEt}_{3}$ was heated over the period of $1.5 \mathrm{~h}$ under reflux temperature. Finally, a purification by column chromatography (petroleum ether: ethyl acetate $=3: 1$ ) gave 3 -(phenylethynyl)quinoline $8 \mathrm{~d}$. Yield $2.290 \mathrm{~g}, 100 \%$, a white solid, m.p. $83-84^{\circ} \mathrm{C} .{ }^{1} \mathrm{H}$ $\operatorname{NMR}\left(600 \mathrm{MHz}, \mathrm{CDCl}_{3}\right): \delta=9.00(\mathrm{~d}, J=2.0 \mathrm{~Hz}, 1 \mathrm{H}, 2-\mathrm{H}), 8.30(\mathrm{~d}, J=2.0$ $\mathrm{Hz}, 1 \mathrm{H}, 4-\mathrm{H}), 8.10(\mathrm{~d}, J=8.3 \mathrm{~Hz}, 1 \mathrm{H}, 8-\mathrm{H}), 7.79(\mathrm{~d}, J=8.3 \mathrm{~Hz}, 1 \mathrm{H}, 5-\mathrm{H})$, 7.72 (ddd, $J=1.5,6.9,8.3 \mathrm{~Hz}, 1 \mathrm{H}, 7-\mathrm{H}), 7.63-7.53\left(\mathrm{~m}, 3 \mathrm{H}, 6-\mathrm{H}, 2^{\prime}-\mathrm{H}, 6^{\prime}-\right.$ H), 7.42-7.35 (m, 3H, 3'-H, 4'-H, 5'-H) ppm. ${ }^{13} \mathrm{C} \mathrm{NMR} \mathrm{(150} \mathrm{MHz,} \mathrm{CDCl} 3$ ): $\delta$ $=152.1(+, \mathrm{C} 2), 146.8(\mathrm{o}, \mathrm{C} 8 \mathrm{a}), 138.3(+, \mathrm{C} 4), 131.8\left(+, \mathrm{C} 2{ }^{\prime}, \mathrm{C}^{\prime}\right), 130.1$ (+, C7), $129.4(+, \mathrm{C} 8), 128.9\left(+, \mathrm{C}^{\prime}\right), 128.5\left(+, \mathrm{C}^{\prime}, \mathrm{C}^{\prime}\right), 127.6(+, \mathrm{C} 6)$, 127.3 (+, C5), 127.3 (o, C4a), 122.6 (o, C1'), 117.5 (o, C3), 92.7 (o, C $\beta$ ), 86.7 (o, C $\alpha$ ) ppm. IR (ATR): 3008, 2162, 1619, 1599, 1564, 1484, 1443, 1409, 1368, 1294, 1196, 1124, 1113, 1072, 980, 905, 860, 784, 756, 691, $656,640,539,515,498,474,429 \mathrm{~cm}^{-1}$. HRMS (ESI): $\mathrm{m} / \mathrm{z}$ calcd for $\mathrm{C}_{17} \mathrm{H}_{11} \mathrm{NNa}[\mathrm{M}+\mathrm{Na}]^{+} 252.0789$, found 252.0796 .

Methyl 4-(quinolin-3-ylethynyl)benzoate (8e): According to Procedure 2

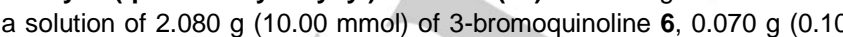
$\mathrm{mmol})$ of $\mathrm{Pd}\left(\mathrm{PPh}_{3}\right)_{2} \mathrm{Cl}_{2}, 0.038 \mathrm{~g}(0.20 \mathrm{mmol}) \mathrm{Cul}$ and $1.680 \mathrm{~g}(10.50 \mathrm{mmol})$ of methyl 4-ethynylbenzoate in $30 \mathrm{~mL}$ of anhydrous $\mathrm{NEt}_{3}$ was heated over the period of $1.5 \mathrm{~h}$ under reflux temperature. Finally, a purification by column chromatography (petroleum ether: ethyl acetate $=3: 1$ ) gave Yield $2.153 \mathrm{~g}, 85 \%$, a brown solid, m.p. $138-139^{\circ} \mathrm{C}$. ${ }^{1} \mathrm{H} \mathrm{NMR}\left(600 \mathrm{MHz}, \mathrm{CDCl}_{3}\right)$ : $\delta=9.00(\mathrm{~d}, J=2.2 \mathrm{~Hz}, 1 \mathrm{H}, 2-\mathrm{H}), 8.33(\mathrm{~d}, J=2.2 \mathrm{~Hz}, 1 \mathrm{H}, 4-\mathrm{H}), 8.11(\mathrm{~d}, J$ $=8.6 \mathrm{~Hz}, 1 \mathrm{H}, 8-\mathrm{H}$ ), 8.06 (ddd, $\left.J=1.4,2.0,8.3 \mathrm{~Hz}, 2 \mathrm{H}, 3^{\prime}-\mathrm{H}, 5^{\prime}-\mathrm{H}\right), 7.81$ (d, $J=8.6 \mathrm{~Hz}, 1 \mathrm{H}, 5-\mathrm{H}$ ), 7.74 (ddd, $J=1.5,6.8,8.6 \mathrm{~Hz}, 1 \mathrm{H}, 7-\mathrm{H}$ ), 7.65 (ddd, $J=1.4,2.0,8.3 \mathrm{~Hz}, 2 \mathrm{H}, 2^{\prime}-\mathrm{H}, 4^{\prime}-\mathrm{H}$ ), 7.58 (ddd, $J=1.5,6.8,8.6 \mathrm{~Hz}, 1 \mathrm{H}, 6-$ $\mathrm{H}), 3.94\left(\mathrm{~s}, 3 \mathrm{H}, \mathrm{COOCH}_{3}\right)$ ppm. ${ }^{13} \mathrm{C} \mathrm{NMR}\left(150 \mathrm{MHz}, \mathrm{CDCl}_{3}\right): \delta=166.5(\mathrm{o}$, COO), 152.0 (+, C2), 147.0 (o, C8a), 138.7 (+, C4), 131.7 (+, C2', C4'), $130.4(+, \mathrm{C} 8), 130.0$ (o, C4'), $129.7\left(+, \mathrm{C}^{\prime}, \mathrm{C} 5^{\prime}\right), 129.5(+, \mathrm{C} 7), 127.7(+$, C5), $127.5(+, C 6), 127.3$ (o, C4a), 127.2 (o, C1'), 116.9 (o, C3), 91.8 (o, $\mathrm{C} \beta), 89.5$ (o, $\mathrm{C \alpha}), 52.3\left(+, \mathrm{CH}_{3}\right)$ ppm. IR (ATR): 3017, 1716, 1276, 1098,
$953,905,855,767,742,697,480 \mathrm{~cm}^{-1}$. Spectroscopic data are in agreement with those reported in the literature. ${ }^{[24]}$

Methyl 2-(quinolin-3-ylethynyl)benzoate (8f): According to Procedure 2, a solution of $2.080 \mathrm{~g}(10.00 \mathrm{mmol})$ of 3-bromoquinoline $6,0.070 \mathrm{~g}(0.10$ $\mathrm{mmol})$ of $\mathrm{Pd}\left(\mathrm{PPh}_{3}\right)_{2} \mathrm{Cl}_{2}, 0.038 \mathrm{~g}(0.20 \mathrm{mmol}) \mathrm{Cul}$ and $1.680 \mathrm{~g}$ (10.50 mmol) of methyl 2-ethynylbenzoate in $30 \mathrm{~mL}$ of anhydrous $\mathrm{NEt}_{3}$ was heated over the period of $1.5 \mathrm{~h}$ under reflux temperature. Finally, a purification by column chromatography (petroleum ether: ethyl acetate $=3: 1$ ) gave Yield $1.607 \mathrm{~g}, 56 \%$, a light brownish solid, m.p. $64-65^{\circ} \mathrm{C} .{ }^{1} \mathrm{H}$ NMR $(600 \mathrm{MHz}$, $\left.\mathrm{CDCl}_{3}\right): \delta=9.05(\mathrm{~d}, J=2.0 \mathrm{~Hz}, 1 \mathrm{H}, 2-\mathrm{H}), 8.36(\mathrm{~d}, J=2.0 \mathrm{~Hz}, 1 \mathrm{H}, 4-\mathrm{H})$, $8.11(\mathrm{~d}, J=8.6 \mathrm{~Hz}, 1 \mathrm{H}, 8-\mathrm{H}), 8.02\left(\mathrm{dd}, J=1.5,7.8 \mathrm{~Hz}, 1 \mathrm{H}, 6^{\prime}-\mathrm{H}\right), 7.81$ (d, $J=8.6 \mathrm{~Hz}, 1 \mathrm{H}, 5-\mathrm{H}), 7.76-7.69\left(\mathrm{~m}, 2 \mathrm{H}, 3^{\prime}-\mathrm{H}, 7-\mathrm{H}\right), 7.60-7.50\left(\mathrm{~m}, 2 \mathrm{H}, 4^{\prime}-\mathrm{H}\right.$ $6-\mathrm{H}), 7.42$ (td, $\left.J=1.3,7.8 \mathrm{~Hz}, 1 \mathrm{H}, 5^{\prime}-\mathrm{H}\right), 3.99\left(\mathrm{~s}, 3 \mathrm{H}, \mathrm{COOCH}_{3}\right) \mathrm{ppm} .{ }^{13} \mathrm{C}$ $\operatorname{NMR}\left(150 \mathrm{MHz}, \mathrm{CDCl}_{3}\right): \delta=166.5$ (o, COO), $152.2(+, \mathrm{C} 2), 147.0$ (o, C8a), $138.5(+, \mathrm{C} 4), 134.2\left(+, \mathrm{C}^{\prime}\right), 131.9\left(\mathrm{o}, \mathrm{C} 2^{\prime}\right), 131.8\left(+, \mathrm{C} 3^{\prime}\right), 130.7\left(+, \mathrm{C} 4{ }^{\prime}\right)$ 130.2(+, C8), $129.5\left(+, C^{\prime}\right), 128.5(+, C 7), 127.7(+, C 5), 127.4(+, C 6)$ 127.3 (C4a), 123.1 (o, C1'), 117.5 (o, C3), 91.5 (o, C 3 ), 91.4 (o, Ca), 52.3 $\left(+, \mathrm{CH}_{3}\right)$ ppm. IR (ATR): 2952, 1731, 1565, 1485, 1435, 1270, 1250, 1111 $1076,899,746,692,471 \mathrm{~cm}^{-1}$. Spectroscopic data are in agreement with those reported in the literature. ${ }^{[24]}$

2,3,4,5,6-Pentaphenyl-1-(quinoline-3-yl)benzene (8g): Benzophenone $(10 \mathrm{~g})$ was melted in a $50 \mathrm{~mL}$ round-bottomed flask fitted with an ai condenser. Corresponding 3-(phenylethynyl)quinoline $8 d$ d $0.458 \mathrm{~g}, 2.00$ $\mathrm{mmol}$ ) and tetraphenylcyclopentadienone $(0.961 \mathrm{~g}, 2.50 \mathrm{mmol})$ were added to the flask, which was heated for $0.5 \mathrm{~h}$ using a heat gun. The solution was cooled to it and toluene $(10 \mathrm{~mL})$ was added to prevent the solidification of the benzophenone. After cooling, $n$-hexane $(50 \mathrm{~mL})$ was added, resulting in the precipitation of a product, which was collected by vacuum filtration. Yield $0.590 \mathrm{~g}, 51 \%$, a white solid, m.p. $334-336{ }^{\circ} \mathrm{C} .{ }^{1} \mathrm{H}$ $\operatorname{NMR}\left(600 \mathrm{MHz}, \mathrm{CDCl}_{3}\right): \delta=8.45\left(\mathrm{~d}, J=2 \mathrm{~Hz}, 1 \mathrm{H}, 2^{\mathrm{i}}-\mathrm{H}\right), 7.84(\mathrm{~d}, J=8.4$ $\mathrm{Hz}, 1 \mathrm{H}, 8 \mathrm{i}-\mathrm{H}), 7.59\left(\mathrm{~d}, J=2 \mathrm{~Hz}, 1 \mathrm{H}, 4^{\mathrm{i}}-\mathrm{H}\right), 7.53-7.51\left(\mathrm{~m}, 1 \mathrm{H}, 7^{\mathrm{i}}-\mathrm{H}\right), 7.43(\mathrm{~d}$, $\left.J=8.0 \mathrm{~Hz}, 5^{\mathrm{i}}-\mathrm{H}\right), 7.35-7.33\left(\mathrm{~m}, 1 \mathrm{H}, 6^{\mathrm{i}}-\mathrm{H}\right), 6.88-6.85\left(\mathrm{~m}, 20 \mathrm{H}, 2^{\mathrm{i}}-\mathrm{H}, 6^{\mathrm{ii}}-\mathrm{H}\right.$

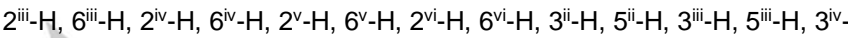
$\left.\mathrm{H}, 5^{\mathrm{iv}}-\mathrm{H}, 3^{\mathrm{v}}-\mathrm{H}, 5^{\mathrm{v}}-\mathrm{H}, 3^{\mathrm{v}}-\mathrm{H}, 5^{\mathrm{vi}}-\mathrm{H}\right), 6.83-6.78\left(\mathrm{~m}, 4 \mathrm{H}, 4^{\mathrm{ii}}-\mathrm{H}, 4^{\mathrm{iii}}-\mathrm{H}, 4^{\mathrm{v}}-\mathrm{H}, 4^{\mathrm{vi}}-\mathrm{H}\right)$, 6.78-6.77 (m, $\left.1 \mathrm{H}, 4^{\text {iv }}-\mathrm{H}\right)$ ppm. ${ }^{13} \mathrm{C}$ NMR $\left(150 \mathrm{MHz}, \mathrm{CDCl}_{3}\right): \delta=152.9(+$, C2i), 145.6 (o, C8a'), 141.4 (o, C4), 141.07 (o, C2, C6), 140.88 (o, C3, C5) 140.44 (o, C1 $\left.{ }^{\mathrm{iv}}\right), 140.34$ (o, C1ii, C1vi), 139.96 (o, C1iii, C1v), 137.8 (+, C4i), 136.51 (o, C1), 134.2 (o, C3'), 131.51/131.48/131.45/131.41/131.37 (+,

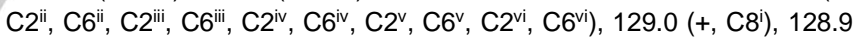
(+, C7i), $127.6\left(+\right.$, C5 $\left.^{\mathrm{i}}\right), 127.4 / 127.0 / 126.85 / 126.83 / 126.78$ (+, C3ii, C5ii,

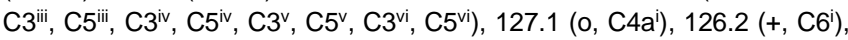

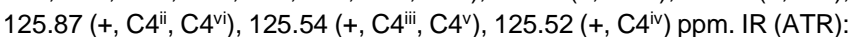
$3055,3024,1662,1600,1495,1440,1401,1354,1311,1275,1261,1127$ $1070,1029,966,906,838,817,779,747,738,719,695,647,638,555$, $544,535,480,443,413 \mathrm{~cm}^{-1}$. HRMS (APCl): $\mathrm{m} / z$ calcd for $\mathrm{C}_{45} \mathrm{H}_{32} \mathrm{~N}[\mathrm{M}+\mathrm{H}]^{+}$ 586.2529 , found 586.2530

General procedure for the preparation of the salts (Procedure 3): Samples of $0.50 \mathrm{mmol}$ of the corresponding esters were dissolved in toluene containing $50 \mu \mathrm{l}$ of nitrobenzene. Then $0.75 \mathrm{mmol}$ of dimethyl sulfate was added with stirring. Thereafter the resulting mixture was stirred under reflux temperature. After completion of the reaction (controlled by TLC), the solution was cooled, the crude product was filtered off, washed with ethyl acetate $(3 \times 10 \mathrm{~mL})$, and dried to afford the product.

1-Methyl-3-phenylquinolinium methylsulfate (9a): According to Procedure 3 , a solution of $0.513 \mathrm{~g}(2.50 \mathrm{mmol})$ of 3-phenylquinoline, $50 \mu \mathrm{l}$ drop of nitrobenzene and $0.284 \mathrm{~mL}(3.00 \mathrm{mmol})$ of dimethyl sulfate in 15 $\mathrm{mL}$ of anhydrous toluene was heated for $2 \mathrm{~h}$ under reflux temperature to give 1-methyl-3-phenylquinolinium methylsulfate $9 a$. Yield $0.796 \mathrm{~g}, 96 \%$, a yellow solid, m.p. $211-212{ }^{\circ} \mathrm{C} .{ }^{1} \mathrm{H}$ NMR $\left(600 \mathrm{MHz},\left[\mathrm{D}_{6}\right] \mathrm{DMSO}\right): \delta=9.96$ (d, $J=1.4 \mathrm{~Hz}, 1 \mathrm{H}, 2-\mathrm{H}), 9.62(\mathrm{~d}, J=1.4 \mathrm{~Hz}, 1 \mathrm{H}, 4-\mathrm{H}), 8.53(\mathrm{~d}, J=8.9 \mathrm{~Hz}, 1 \mathrm{H}$, 
8-H), 8.49 (d, $J=8.9 \mathrm{~Hz}, 1 \mathrm{H}, 5-\mathrm{H}), 8.27$ (ddd, $J=1.5,7.1,8.9 \mathrm{~Hz}, 1 \mathrm{H}, 7-$ H), 8.08 (ddd, $J=1.5,7.1,8.9 \mathrm{~Hz}, 1 \mathrm{H}, 6-\mathrm{H}), 8.04-8.03\left(\mathrm{~m}, 2 \mathrm{H}, 2^{\prime}-\mathrm{H}, 6^{\prime}-\mathrm{H}\right.$ ), 7.66 (t, $\left.2 \mathrm{H}, J=7.62 \mathrm{~Hz}, 3^{\prime}-\mathrm{H}, 5^{\prime}-\mathrm{H}\right), 7.60-7.58\left(\mathrm{~m}, 1 \mathrm{H}, 4^{\prime}-\mathrm{H}\right), 4.72(\mathrm{~s}, 3 \mathrm{H}$, $\mathrm{NCH}_{3}$ ), 3.37 (s, 3H, $\left.\mathrm{CH}_{3} \mathrm{SO}_{4}\right)$ ppm. ${ }^{13} \mathrm{C} \mathrm{NMR} \mathrm{(150} \mathrm{MHz,} \mathrm{[D6]DMSO):} \delta=$ 149.3 (+, C2), 142.9 (+, C4), 137.2 (o, C8a), 135.2 (+, C7), 133.5 (o, C3), 133.4 (o, C1'), $130.5(+, C 5), 130.2(+, C 6), 129.8\left(+, C 4^{\prime}\right), 129.5\left(+, C 3^{\prime}\right.$, $\left.\mathrm{C}^{\prime}\right), 129.2$ (o, C4a), $127.4\left(+, \mathrm{C}^{\prime}, \mathrm{C}^{\prime}\right), 119.0(+, \mathrm{C} 8), 52.8\left(+, \mathrm{CH}_{3} \mathrm{SO}_{4}\right)$, $45.4\left(+, \mathrm{NCH}_{3}\right)$ ppm. IR (ATR): 3052, 1534, 1504, 1362, 1206, 1051, 1006, 859, 773, 733, 695, 607, 576, 496, 457, $427 \mathrm{~cm}^{-1}$. MS (ESI): $\mathrm{m} / z=220.1$ [M]+. HRMS (ESI): $\mathrm{m} / z$ calcd for $\mathrm{C}_{16} \mathrm{H}_{14} \mathrm{~N}[\mathrm{M}]^{+} 220.1121$, found 220.1126 .

1-Methyl-3-(naphthalen-1-yl)quinolinium methylsulfate (9b): According to Procedure 3 , a solution of $0.638 \mathrm{~g}(2.50 \mathrm{mmol})$ of 3 (naphthalen-1-yl)quinoline, $50 \mu \mathrm{l}$ drop of nitrobenzene and $0.284 \mathrm{~mL}(3.00$ $\mathrm{mmol}$ ) of dimethyl sulfate in $15 \mathrm{~mL}$ of anhydrous toluene was heated for 2 $\mathrm{h}$ under reflux temperature to give 1-methyl-3-(naphthalen-1yl)quinolinium methylsulfate $9 \mathrm{~b}$. Yield $0.916 \mathrm{~g}, 96 \%$, a yellow solid, m.p. 219-220 ${ }^{\circ} \mathrm{C} .{ }^{1} \mathrm{H}$ NMR $\left(600 \mathrm{MHz},\left[\mathrm{D}_{6}\right] \mathrm{DMSO}\right): \delta=9.82(\mathrm{~d}, J=1.3 \mathrm{~Hz}, 1 \mathrm{H}$, 2-H), $9.47(\mathrm{~s}, 1 \mathrm{H}, 4-\mathrm{H}), 8.61(\mathrm{~d}, J=8.9 \mathrm{~Hz}, 1 \mathrm{H}, 8-\mathrm{H}), 8.54(\mathrm{~d}, J=8.2 \mathrm{~Hz}$, $1 \mathrm{H}, 5-\mathrm{H}$ ), 8.35 (ddd, $J=1.5,7.2,8.8 \mathrm{~Hz}, 1 \mathrm{H}, 7-\mathrm{H}), 8.19$ (t, $J=4.7 \mathrm{~Hz}, 1 \mathrm{H}$ $\left.3^{\prime}-\mathrm{H}\right), 8.15-8.12\left(\mathrm{~m}, 2 \mathrm{H}, 6-\mathrm{H}, 5^{\prime}-\mathrm{H}\right), 7.95\left(\mathrm{~d}, J=8.4 \mathrm{~Hz}, 1 \mathrm{H}, 8^{\prime}-\mathrm{H}\right), 7.76-$ $7.75\left(\mathrm{~m}, 2 \mathrm{H}, 2^{\prime}-\mathrm{H}, 4^{\prime}-\mathrm{H}\right), 7.67$ (ddd, $\left.J=1.2,6.9,8.1 \mathrm{~Hz}, 1 \mathrm{H}, 6^{\prime}-\mathrm{H}\right), 7.62$ (ddd, $\left.J=1.2,6.9,8.1 \mathrm{~Hz}, 1 \mathrm{H}, 7^{\prime}-\mathrm{H}\right), 4.73\left(\mathrm{~s}, 1 \mathrm{H}, \mathrm{NCH}_{3}\right), 3.36(\mathrm{~s}, 3 \mathrm{H}$, $\left.\mathrm{CH}_{3} \mathrm{SO}_{4}\right)$ ppm. ${ }^{13} \mathrm{C} \mathrm{NMR}\left(150 \mathrm{MHz},\left[\mathrm{D}_{6}\right] \mathrm{DMSO}\right): \delta=151.1(+, \mathrm{C} 2), 146.6$ (+, C4), 137.5 (o, C8a), 135.4 (+, C7), 133.4 (o, C3), 132.3 (o, C1'), 130.6 (o, C8a'), $\left.130.5(+, \mathrm{C} 5), 130.2(+, \mathrm{C} 6), 129.9(+, \mathrm{C} 3)^{\prime}\right), 129.2(+, \mathrm{C} 4 \mathrm{a}), 128.9$ (+, C4'), $128.6\left(+, \mathrm{C}^{\prime}\right), 127.5\left(+, \mathrm{C} 7^{\prime}\right), 126.7\left(+, \mathrm{C6}^{\prime}\right), 125.7\left(+, \mathrm{C} 2^{\prime}\right), 124.7$ (+, C8'), $119.0(+, \mathrm{C} 8), 52.8\left(+, \mathrm{CH}_{3} \mathrm{SO}_{4}\right), 45.3\left(+, \mathrm{NCH}_{3}\right)$ ppm. IR (ATR): 3041, 2935, 2829, 1524, 1383, 1247, 1215, 1060, 1011, 807, 774, 738, $650,609,579,576,551,483,433 \mathrm{~cm}^{-1}$. MS (ESI): $\mathrm{m} / z=270.1$ [M] $^{+}$. HRMS (ESI): $m / z$ calcd for $\mathrm{C}_{20} \mathrm{H}_{16} \mathrm{~N}[\mathrm{M}]^{+} 270.1283$, found 270.1285 .

1-Methyl-3-(phenanthren-9-yl)quinolinium methylsulfate (9c) According to Procedure 3 , a solution of $0.638 \mathrm{~g}(2.50 \mathrm{mmol})$ of 3 (phenanthren-9-yl)quinoline, $50 \mu \mathrm{l}$ of nitrobenzene and $0.284 \mathrm{~mL}(3.00$ $\mathrm{mmol}$ ) of dimethyl sulfate in $15 \mathrm{~mL}$ of anhydrous toluene was heated for 2 $h$ under reflux temperature to give 1-methyl-3-(phenanthren-9yl)quinolinium methylsulfate 9c. Yield $1.046 \mathrm{~g}, 97 \%$, a yellow solid, m.p. 209-210 ${ }^{\circ} \mathrm{C}$. ${ }^{1} \mathrm{H}$ NMR $\left(600 \mathrm{MHz},\left[\mathrm{D}_{6}\right] \mathrm{DMSO}\right): \delta=9.89(\mathrm{~d}, J=1.1 \mathrm{~Hz}, 1 \mathrm{H}$ 2-H), $9.54(\mathrm{~s}, 1 \mathrm{H}, 4-\mathrm{H}), 9.05\left(\mathrm{~d}, J=8.3 \mathrm{~Hz}, 1 \mathrm{H}, 5^{\prime}-\mathrm{H}\right), 8.98(\mathrm{~d}, J=8.2 \mathrm{~Hz}$, $\left.1 \mathrm{H}, 4^{\prime}-\mathrm{H}\right), 8.63(\mathrm{~d}, J=9.2 \mathrm{~Hz}, 1 \mathrm{H}, 8-\mathrm{H}), 8,56(\mathrm{~d}, J=8.2 \mathrm{~Hz}, 1 \mathrm{H}, 5-\mathrm{H}), 8.37$ (ddd, $J=1.5,7.2,8.8 \mathrm{~Hz}, 1 \mathrm{H}, 7-\mathrm{H}), 8.16-8.11\left(\mathrm{~m}, 3 \mathrm{H}, 6-\mathrm{H}, 1^{\prime}-\mathrm{H}, 10^{\prime}-\mathrm{H}\right)$ $7.98\left(\mathrm{~d}, J=7.6 \mathrm{~Hz}, 1 \mathrm{H}, 8^{\prime}-\mathrm{H}\right), 7.86-7.83\left(\mathrm{~m}, 2 \mathrm{H}, 3^{\prime}-\mathrm{H}, 6^{\prime}-\mathrm{H}\right), 7.78(\mathrm{~m}, 1 \mathrm{H}$, 2'-H), 7.71 (ddd, $\left.J=0.9,7.2,8.0 \mathrm{~Hz}, 1 \mathrm{H}, 7^{\prime}-\mathrm{H}\right), 4.74\left(\mathrm{~s}, 3 \mathrm{H}, \mathrm{NCH}_{3}\right), 3.36$ (s, $\left.3 \mathrm{H}, \mathrm{CH}_{3} \mathrm{SO}_{4}\right)$ ppm. ${ }^{13} \mathrm{C} \mathrm{NMR}\left(150 \mathrm{MHz},\left[\mathrm{D}_{6}\right] \mathrm{DMSO}\right): \delta=151.1(+, \mathrm{C} 2)$, 146.7 (+, C4), 137.7 (o, C8a), 135.5 (+, C7), 133.5 (o, C3), 131.1 (o, C9'), 130.6 (o, C10a'), $130.5(+, \mathrm{C} 5), 130.2(+, \mathrm{C} 6), 130.2$ (o, C4b'), 130.1 (o, C4a'), 130.0 (+, C10 '), 129.5 (o, C8a'), 129.2 (o, C4a), 129.1 (+, C1'), $128.2\left(+, 3^{\prime}\right), 127.7\left(+, C 2^{\prime}\right), 127.6\left(+, C 7^{\prime}\right), 127.6\left(+, C 6^{\prime}\right), 125.9\left(+, C 8^{\prime}\right)$, 123.7 (+, C5), $123.1\left(+, \mathrm{C}^{\prime}\right), 119.0(+, \mathrm{C} 8), 52.8\left(+, \mathrm{CH}_{3} \mathrm{SO}_{4}\right), 45.3(+$ $\mathrm{NCH}_{3}$ ) ppm. IR (ATR): 3042, 2944, 1627, 1607, 1578, 1525, 1495, 1447, 1378, 1316, 1217, 1059, 1010, 900, 825, 726, 657, 608, 577, $426 \mathrm{~cm}^{-1}$. HRMS (ESI): $\mathrm{m} / z$ calcd for $\mathrm{C}_{24} \mathrm{H}_{18} \mathrm{~N}[\mathrm{M}]^{+} 320.1434$, found 320.1435 .

\section{1-Methyl-3-(phenanthren-9-yl)quinolinium hexafluorophosphate} $\left(9 \mathrm{CPF}_{6}\right)$ : Methylsulfate salt $9 \mathrm{c}(0.431 \mathrm{~g}, 1.00 \mathrm{mmol})$ was dissolved in water, then $\mathrm{NH}_{4} \mathrm{PF}_{6}$ (1.05 equiv) in water was added to the prepared solution Hexafluorophosphate salt precipitated immediately. After $0.5 \mathrm{~h}$ the crude product was filtered off and washed with water $(3 \times 30 \mathrm{~mL})$ and ethyl acetate $(3 \times 30 \mathrm{~mL})$, dried in vacuo to give $9 \mathrm{CPF}_{6}$. Yield $0.460 \mathrm{~g}, 99 \%$, a yellow solid, m.p. $223-225^{\circ} \mathrm{C}$ (decomp.). ${ }^{1} \mathrm{H}$ NMR $\left(600 \mathrm{MHz}, \mathrm{CD}_{3} \mathrm{CN}\right): \delta=$ $9.38(\mathrm{~d}, J=1.1 \mathrm{~Hz}, 1 \mathrm{H}), 9.23(\mathrm{~s}, 1 \mathrm{H}), 8.90(\mathrm{~d}, J=8.5 \mathrm{~Hz}, 1 \mathrm{H}), 8.83(\mathrm{~d}, J=$ $8.3 \mathrm{~Hz}, 1 \mathrm{H}), 8.45-8.43(\mathrm{~m}, 1 \mathrm{H}), 8.40-8.38(\mathrm{~m}, 1 \mathrm{H}), 8.28$ (ddd, $J=1.5,7.2$, $8.9 \mathrm{~Hz}, 1 \mathrm{H}), 8.07-8.03(\mathrm{~m}, 2 \mathrm{H}), 7.99(\mathrm{~s}, 1 \mathrm{H}), 7.90-7.88(\mathrm{~m}, 1 \mathrm{H}), 7.82-7.79$ (m, 2H), 7.72 (ddd, $J=1.0,7.0,7.9 \mathrm{~Hz}, 1 \mathrm{H}$ ), 7.67 (ddd, $J=1.2,6.9,8.2$ $\mathrm{Hz}, 1 \mathrm{H}) 4.65\left(\mathrm{~s}, 3 \mathrm{H}, \mathrm{NCH}_{3}\right) \mathrm{ppm} .{ }^{13} \mathrm{C} \mathrm{NMR}\left(150 \mathrm{MHz}, \mathrm{CD}_{3} \mathrm{CN}\right): \delta=151.7$ (+), $148.3(+), 139.0$ (o), $136.9(+), 135.2$ (o), 131.9 (o), 131.8 (o), 131.62 $(+), 131.60(0), 131.58(+), 131.5(+), 131.3(+), 130.64(0), 130.63(0)$ $130.2(+), 129.2(+), 128.65(+), 128.61(+), 128.60(+), 126.7(+), 124.6$ $(+), 124.6(+), 123.9(+), 119.6(+), 46.6(+)$ ppm. IR (ATR): 3053, 1525 $1447,1378,1354,1247,1214,1172,1060,1012,901,825,746,724,658$, $608,577,551,490,427 \mathrm{~cm}^{-1}$. HRMS (ESI): $\mathrm{m} / z$ calcd for $\mathrm{C}_{24} \mathrm{H}_{18} \mathrm{~N}[\mathrm{M}]^{+}$ 320.1434 , found 320.1440

1-Methyl-3-(phenylethynyl)quinolinium methylsulfate (9d): According to Procedure 3, a solution of $1.145 \mathrm{~g} \quad(5.00 \mathrm{mmol})$ of 3 (phenylethynyl)quinoline $8 \mathrm{~d}, 1$ drop of nitrobenzene and $0.711 \mathrm{~mL}(7.50$ $\mathrm{mmol}$ ) of dimethyl sulfate in $25 \mathrm{~mL}$ of anhydrous toluene was heated over the period of $1.5 \mathrm{~h}$ under reflux temperature to give 1-methyl-3(phenylethynyl)quinolinium methyl-sulfate $9 \mathrm{~d}$. Yield $1.757 \mathrm{~g}, 99 \%$, a yellow solid, m.p. $224-225^{\circ} \mathrm{C}$ (decomp.). ${ }^{1} \mathrm{H}$ NMR $(600 \mathrm{MHz}$, [D6]DMSO): $\delta=9.87$ (s, $1 \mathrm{H}, 2-\mathrm{H}), 9.50(\mathrm{~s}, 1 \mathrm{H}, 4-\mathrm{H}), 8.54(\mathrm{~d}, J=8.8 \mathrm{~Hz}, 1 \mathrm{H}, 8-\mathrm{H}), 8.45(\mathrm{~d}, J=$ $8.8 \mathrm{~Hz}, 1 \mathrm{H}, 5-\mathrm{H}$ ), 8.31 (ddd, $J=1.3,7.1,8.8 \mathrm{~Hz}, 1 \mathrm{H}, 7-\mathrm{H}$ ), 8.10 (t, $J=8.8$ $\mathrm{Hz}, 1 \mathrm{H}, 6-\mathrm{H}), 7.74-7.66\left(\mathrm{~m}, 2 \mathrm{H}, 2^{\prime}-\mathrm{H}, 6^{\prime}-\mathrm{H}\right), 7.60-7.50\left(\mathrm{~m}, 3 \mathrm{H}, 3^{\prime}-\mathrm{H}, 4^{\prime}-\mathrm{H}\right.$, $\left.5^{\prime}-\mathrm{H}\right), 4.66\left(\mathrm{~s}, 3 \mathrm{H}, \mathrm{NCH}_{3}\right), 3.38\left(\mathrm{~s}, 3 \mathrm{H}, \mathrm{CH}_{3} \mathrm{SO}_{4}\right) \mathrm{ppm} .{ }^{13} \mathrm{C} \mathrm{NMR}(150 \mathrm{MHz}$ [D6]DMSO): $\delta=153.1(+, \mathrm{C} 2), 147.9(+, \mathrm{C} 4), 137.3$ (o, C8a), $136.0(+, \mathrm{C} 7)$, $131.7\left(+, 2^{\prime}, \mathrm{C}^{\prime}\right), 130.6\left(+, \mathrm{C} 4^{\prime}\right), 130.3(+, \mathrm{C} 6), 130.2(+, \mathrm{C} 5), 129.1(+$ C3', C5'), 128.7 (o, C4a), 120.5 (o, C1'), 119.2 (+, C8), 116.7 (o, C3), 94.8 $(\mathrm{o}, \mathrm{C} \beta), 83.3(\mathrm{o}, \mathrm{C} \alpha), 52.8\left(+, \mathrm{CH}_{3} \mathrm{SO}_{4}\right), 45.4\left(+, \mathrm{NCH}_{3}\right) \mathrm{ppm}$. IR (ATR): $3043,2941,2223,1631,1606,1578,1520,1493,1494,1444,1365,1227$, $1210,1173,1140,1059,1010,929,910,878,766,737,695,607,577$ $552,524,501,479,429 \mathrm{~cm}^{-1}$. MS (ESI): $\mathrm{m} / z=244.1$ [M]'. HRMS (ESI): $\mathrm{m} / \mathrm{z}$ calcd for $\mathrm{C}_{18} \mathrm{H}_{14} \mathrm{~N}[\mathrm{M}]^{+}$244.1121, found 244.1126.

3-((4-(Methoxycarbonyl)phenyl)ethynyl)-1-methylquinolinium methylsulfate (9e): According to Procedure 3 , a solution of $1.435 \mathrm{~g} \mathrm{(5.00}$ $\mathrm{mmol}$ ) of methyl 4-(quinolin-3-ylethynyl)benzoate $8 \mathrm{e}, 50 \mu \mathrm{l}$ of nitrobenzene and $0.711 \mathrm{~mL}(7.50 \mathrm{mmol})$ of dimethyl sulfate in $25 \mathrm{~mL}$ of anhydrous toluene was heated for $1.5 \mathrm{~h}$ under reflux temperature to give 3-(4(methoxycarbonyl)phenyl)ethynyl)-1-methylquinolinium methylsulfate $9 \mathrm{e}$. Yield 2.044 g, 99\%, a brownish solid, m.p. $219-220^{\circ} \mathrm{C}$. ${ }^{1} \mathrm{H}$ NMR $(600 \mathrm{MHz}$, [D6]DMSO): $\delta=9.91(\mathrm{~d}, J=1.2 \mathrm{~Hz}, 1 \mathrm{H}, 2-\mathrm{H}), 9.54(\mathrm{~d}, J=1.2 \mathrm{~Hz}, 1 \mathrm{H}, 4$ H), $8.55(\mathrm{~d}, J=9.0 \mathrm{~Hz}, 1 \mathrm{H}, 8-\mathrm{H}), 8.46(\mathrm{~d}, J=9.0 \mathrm{~Hz}, 1 \mathrm{H}, 5-\mathrm{H}), 8.33$ (ddd, $J=1.3,7.0,9.0 \mathrm{~Hz}, 1 \mathrm{H}, 6-\mathrm{H}), 8.14-8.07\left(\mathrm{~m}, 3 \mathrm{H}, 7-\mathrm{H}, 2^{\prime}-\mathrm{H}, 6^{\prime}-\mathrm{H}\right), 7.83$ (ddd, $\left.J=1.5,1.9,8.2 \mathrm{~Hz}, 2 \mathrm{H}, 3^{\prime}-\mathrm{H}, 5^{\prime}-\mathrm{H}\right), 4.66\left(\mathrm{~s}, 3 \mathrm{H}, \mathrm{NCH}_{3}\right), 3.90(\mathrm{~s}, 3 \mathrm{H}$, $\left.\mathrm{COOCH}_{3}\right), 3.37$ (s, $\left.3 \mathrm{H}, \mathrm{CH}_{3} \mathrm{SO}_{4}\right)$ ppm. ${ }^{13} \mathrm{C} \mathrm{NMR}\left(150 \mathrm{MHz},\left[\mathrm{D}_{6}\right] \mathrm{DMSO}\right): \delta$ $=165.5$ (o, COO), $152.2(+$, C2), $148.3(+$, C4), 137.5 (o, C8a), $136.3(+$, C7), $132.1\left(+\right.$, C2' $\left.^{\prime}, \mathrm{C6}^{\prime}\right), 130.7$ (+, C6), 130.5 (o, C4a), 130.4 (+, C5), 129.7 (+, C3', C5'), 128.7 (o, C4'), $125.2\left(\mathrm{C}^{\prime}\right), 119.3(+, \mathrm{C} 8), 116.2$ (o, C3), 93.5 (o, $\mathrm{C} \alpha), 85.9(\mathrm{o}, \mathrm{C} \beta), 52.8\left(+, \mathrm{CH}_{3} \mathrm{SO}_{4}\right), 52.5\left(+, \mathrm{COOCH}_{3}\right), 45.5\left(+, \mathrm{NCH}_{3}\right)$ ppm. IR (ATR): 1721, 1278, 1240, 1215, 1170, 1097, 1057, 1005, 866, 766, $729,696,607,574 \mathrm{~cm}^{-1}$. Spectroscopic data are in agreement with those reported in the literature. ${ }^{[24]}$

\section{3-((2-(Methoxycarbonyl)phenyl)ethynyl)-1-methylquinolinium}

methylsulfate (9f): According to Procedure 3 , a solution of $1.435 \mathrm{~g} \mathrm{(5.00}$ $\mathrm{mmol}$ ) of methyl 2-(quinolin-3-ylethynyl)benzoate $8 \mathbf{f}, 50 \mu \mathrm{l}$ of nitrobenzene and $0.711 \mathrm{~mL}(7.50 \mathrm{mmol})$ of dimethyl sulfate in $25 \mathrm{~mL}$ of anhydrous toluene was heated for $1.5 \mathrm{~h}$ under reflux temperature to give 3-((2(methoxycarbonyl)phenyl)ethynyl)-1-methylquinolinium methylsulfate 9 ff Yield $2.044 \mathrm{~g}$, 99\%, a yellow solid, m.p. $217-218{ }^{\circ} \mathrm{C}$. ${ }^{1} \mathrm{H}$ NMR $(600 \mathrm{MHz}$, [D6]DMSO): $\delta=9.80(\mathrm{~d}, J=1.0 \mathrm{~Hz}, 1 \mathrm{H}, 2-\mathrm{H}), 9.47(\mathrm{~d}, J=1.0 \mathrm{~Hz}, 1 \mathrm{H}, 4-$ H), $8.54(\mathrm{~d}, J=9.1 \mathrm{~Hz}, 1 \mathrm{H}, 8-\mathrm{H}), 8.50(\mathrm{~d}, J=9.1 \mathrm{~Hz}, 1 \mathrm{H}, 5-\mathrm{H}), 8.32$ (ddd $J=1.5,7.1,9.1 \mathrm{~Hz}, 1 \mathrm{H}, 6-\mathrm{H}$ ), 8.11 (ddd, $J=1.5,7.1,9.1 \mathrm{~Hz}, 1 \mathrm{H}, 7-\mathrm{H}$ ) $8.04\left(\mathrm{dd}, J=1.0,8.0 \mathrm{~Hz}, 1 \mathrm{H}, 6^{\prime}-\mathrm{H}\right), 7.85\left(\mathrm{dd}, J=1.0,8.0 \mathrm{~Hz}, 1 \mathrm{H}, 3^{\prime}-\mathrm{H}\right)$, $7.76\left(\mathrm{td}, J=1.5,7.6 \mathrm{~Hz}, 1 \mathrm{H}, 4^{\prime}-\mathrm{H}\right), 7.66\left(\mathrm{td}, J=1.5,7.6 \mathrm{~Hz}, 1 \mathrm{H}, 5^{\prime}-\mathrm{H}\right), 4.66$ (s, 3H, NCH 3$), 3.96\left(\mathrm{~s}, 3 \mathrm{H}, \mathrm{COOCH}_{3}\right), 3.36\left(\mathrm{~s}, \mathrm{CH}_{3} \mathrm{SO}_{4}\right) \mathrm{ppm} .{ }^{13} \mathrm{C} \mathrm{NMR}$ $\left(150 \mathrm{MHz},\left[\mathrm{D}_{6}\right] \mathrm{DMSO}\right): \delta=165.5(\mathrm{o}, \mathrm{COO}), 151.9(+, \mathrm{C} 2), 148.0(+, \mathrm{C} 4)$, 
137.4 (o, C8a), $136.1(+, \mathrm{C} 7), 134.3\left(+, \mathrm{C}^{\prime}\right), 132.7\left(+, \mathrm{C6}{ }^{\prime}\right), 131.8$ (o, C2'), $130.6(+, \mathrm{C} 6), 130.5\left(+, 4^{\prime}\right), 130.4(+, \mathrm{C} 5), 130.2\left(+, \mathrm{C}^{\prime}\right), 128.8$ (o, C4a), 120.7 (o, C1'), 119.2 (+, C8), 116.8 (o, C3), 93.4 (o, Ca), 87.4 (o, C 3 ), 52.8 $\left(+, \mathrm{CH}_{3} \mathrm{SO}_{4}\right), 52.5\left(+, \mathrm{COOCH}_{3}\right), 45.5\left(+, \mathrm{NCH}_{3}\right)$ ppm. IR (ATR): 2923, $2219,1737,1272,1258,1133,1082,832,765,761,698,556,434 \mathrm{~cm}^{-1}$. Spectroscopic data are in agreement with those reported in the literature. ${ }^{[24]}$

\section{1-Methyl-3-(4',5',6'-triphenyl-[1,1':2',1'--terphenyl]-3'-yl)quinolinium} methylsulfate (9g): According to Procedure 3 , a solution of $0.100 \mathrm{~g}(0.171$ $\mathrm{mmol}$ ) of 2,3,4,5,6 pentaphenyl-1-(quinoline-3-yl)benzene, $50 \mu \mathrm{l}$ of nitrobenzene and $0.06 \mathrm{~mL}(0.63 \mathrm{mmol})$ of dimethyl sulfate in $5 \mathrm{~mL}$ of anhydrous toluene was heated for $2 \mathrm{~h}$ under reflux temperature to give 1 methyl-3-(4',5',6'-triphenyl-[1,1':2',1"-terphenyl]-3'-yl)quinolinium

methylsulfate $9 \mathrm{~g}$. Yield $0.122 \mathrm{~g}, 99 \%$, a light green solid, m.p. $321-323^{\circ} \mathrm{C}$ (decomp.). ${ }^{1} \mathrm{H} \mathrm{NMR}\left(600 \mathrm{MHz},\left[\mathrm{D}_{6}\right] \mathrm{DMSO}\right): \delta=9.35$ (d, $J=1.4 \mathrm{~Hz}, 1 \mathrm{H}, 2$ $\mathrm{H}), 8.84(\mathrm{~s}, 1 \mathrm{H}, 4-\mathrm{H}), 8.24(\mathrm{~d}, J=8.1 \mathrm{~Hz}, 1 \mathrm{H}, 8-\mathrm{H}), 8.14$ (ddd, $J=1.5,7.1$, $8.8 \mathrm{~Hz}, 1 \mathrm{H}, 7-\mathrm{H}), 8.06(\mathrm{dd}, J=1.1,8.4 \mathrm{~Hz}, 1 \mathrm{H}, 5-\mathrm{H}), 7.92-7.90(\mathrm{~m}, 1 \mathrm{H}, 6-$ $\mathrm{H}), 7.05(\mathrm{~d}, J=7.7 \mathrm{~Hz}, 2 \mathrm{H}, \mathrm{Ph}), 7.02(\mathrm{~d}, J=7.7 \mathrm{~Hz}, 2 \mathrm{H}, \mathrm{Ph}), 6.96-6.82(\mathrm{~m}$, $21 \mathrm{H}, \mathrm{Ph}), 4.34\left(\mathrm{~s}, 3 \mathrm{H}, \mathrm{NCH}_{3}\right), 3.37\left(\mathrm{~s}, 3 \mathrm{H}, \mathrm{CH}_{3} \mathrm{SO}_{4}\right) \mathrm{ppm} .{ }^{13} \mathrm{C}$ NMR $(150$ $\mathrm{MHz}$, [D6]DMSO): $\delta=150.6(+, \mathrm{C} 2), 147.5(+, \mathrm{C} 4), 142.3(\mathrm{o}), 140.7$ (o), 140.5 (o), 139.3 (o), 139.0 (o), 138.4 (o), 135.53 (o, C8a), 135.48 (+, C7), 134.4 (o, C3), 132.8 (o, C1'), $131.0(+), 130.7(+), 130.64(+), 130.60(+)$, $130.54(+), 130.5(+$, C6), $129.9(+$, C5), 127.7 (o, C4a), 127.4 (o), 126.89 $(+), 126.87(+), 126.80(+), 126.77(+), 126.4(+), 125.92(+), 125.85(+)$, $118.9(+, \mathrm{C} 8), 52.8\left(+, \mathrm{CH}_{3} \mathrm{SO}_{4}\right), 44.8\left(+, \mathrm{NCH}_{3}\right)$ ppm. IR (ATR): 3051, 3023, 2961, 1600, 1524, 1496, 1442, 1378, 1254, 1215, 1063, 1012, 760, $697,618,577,558,441 \mathrm{~cm}^{-1}$. HRMS $(\mathrm{ESI}): \mathrm{m} / z$ calcd for $\mathrm{C}_{46} \mathrm{H}_{34} \mathrm{~N}[\mathrm{M}]^{+}$ 600.2686 , found 600.2687 .

\begin{abstract}
1-Methyl-3-(phenanthren-9-yl)quinolin-2(1H)-one (10): Under a nitrogen atmosphere, 1-methyl-3-(phenanthren-9-yl)quinolinium methylsulfate $(0.431 \mathrm{~g}, 1.00 \mathrm{mmol})$, sulfur $(0.128 \mathrm{~g}, 4.00 \mathrm{mmol})$ and $\mathrm{LiOH}$ $(0.192 \mathrm{~g}, 8.00 \mathrm{mmol})$ were suspended in absolute toluene $(10 \mathrm{~mL})$, and then potassium tert butoxide $(0.896 \mathrm{~g}, 8.00 \mathrm{mmol})$ was added slowly with stirring. The resulting mixture was stirred for $6 \mathrm{~h}$ under reflux at $120^{\circ} \mathrm{C}$. After that, the mixture was concentrated in vacuo, and the crude product was purified by column chromatography with $\mathrm{PE}-\mathrm{EE}$ as an eluent to give 1 methyl-3-(phenanthren-9-yl)quinolin-2(1H)-one 10 . Yield $0.184 \mathrm{~g}, 55 \%$, a white solid, m.p. $56-57^{\circ} \mathrm{C}\left(55-58{ }^{\circ} \mathrm{C}\right) .{ }^{[35]}{ }^{1} \mathrm{H}$ NMR $\left(600 \mathrm{MHz}, \mathrm{CDCl}_{3}\right): \delta=$ $8.77\left(\mathrm{~d}, J=8.3 \mathrm{~Hz}, 1 \mathrm{H}, 5^{\prime}-\mathrm{H}\right), 8.72\left(\mathrm{~d}, J=8.0 \mathrm{~Hz}, 1 \mathrm{H}, 4^{\prime}-\mathrm{H}\right), 790(\mathrm{~s}, 1 \mathrm{H}, 4-$ H), 7.88 (dd, $\left.J=1.4,7.8 \mathrm{~Hz}, 1 \mathrm{H}, 1^{\prime}-\mathrm{H}\right), 7.77-7.76\left(\mathrm{~m}, 2 \mathrm{H}, 5^{\prime}-\mathrm{H}, 10-\mathrm{H}\right), 7.68$ (ddd, $\left.J=1.4,6.9,8.3 \mathrm{~Hz}, 1 \mathrm{H}, 3^{\prime}-\mathrm{H}\right), 7.66-7.64(\mathrm{~m}, 2 \mathrm{H}, 5-\mathrm{H}, 7-\mathrm{H}), 7.60$ (ddd, $J=1.0,6.9,7.8 \mathrm{~Hz}, 1 \mathrm{H}, 2^{\prime}-\mathrm{H}$ ), 7.53 (ddd, $J=1.2,6.9,8.2 \mathrm{~Hz}, 1 \mathrm{H}, 7^{\prime}-\mathrm{H}$ ) $7.49(\mathrm{~d}, J=9.0 \mathrm{~Hz}, 1 \mathrm{H}, 8-\mathrm{H}), 7.32-7.30(\mathrm{~m}, 1 \mathrm{H}, 6-\mathrm{H}), 3.86\left(\mathrm{~s}, 3 \mathrm{H}, \mathrm{NCH}_{3}\right)$ ppm. ${ }^{13} \mathrm{C}$ NMR $\left(150 \mathrm{MHz}, \mathrm{CDCl}_{3}\right): \delta=162.0(+, \mathrm{C} 2), 140.3$ (o, C8a), 139.2 (+, C4), 134.3 (o, C9'), 133.3 (o, C3), 131.7 (o, C10a'), 130.8 (o, C4a'), $130.7(+, C 7), 130.6$ (o, C4b'), $129.1(+, C 5), 128.9\left(+, C 1^{\prime}\right), 128.4\left(+, C 10^{\prime}\right)$, $127.0\left(+, C 3^{\prime}\right), 126.79\left(+, C 8^{\prime}\right), 126.77\left(+, C 2^{\prime}\right), 126.73\left(+, C 7^{\prime}\right), 126.68(+$, $\left.\mathrm{C}^{\prime}\right), 123.1\left(+, \mathrm{C}^{\prime}\right), 122.7\left(+, 4^{\prime}\right), 122.5(+, \mathrm{C} 6), 120.8(\mathrm{o}, \mathrm{C} 4 \mathrm{a}), 114.4(+$, C8), $30.2\left(+, \mathrm{NCH}_{3}\right)$ ppm. IR (ATR): $3059,1638,1590,1570,1528,1495$, $1463,1448,1432,1416,1383,1364,1343,1329,1317,1295,1275,1242$, $1211,1164,1146,1127,1105,1085,1040,1018,1001,979,951,927$, $918,899,884,861,853,805,788,769,753,748,732,721,710,660,620$, $615,578,558,543,509,496,482,463,454,426,416,412,407 \mathrm{~cm}^{-1} . \mathrm{MS}$ (ESI): $m / z=336.0[\mathrm{M}+\mathrm{H}]^{+}$.
\end{abstract}

General procedure for the preparation of thiones (Procedure 4): Under a nitrogen atmosphere the corresponding salt $(1.00 \mathrm{mmol})$, sulfur $(4.00 \mathrm{mmol})$ and $\mathrm{LiOH}(8.00 \mathrm{mmol})$ were suspended in absolute toluene $(10 \mathrm{~mL})$, and then potassium tert-pentoxide $(1.20 \mathrm{mmol}, 1.7 \mathrm{M}$ solution in toluene) was added slowly with stirring. The resulting mixture was stirred for $6 \mathrm{~h}$ under reflux at $120^{\circ} \mathrm{C}$. After the mixture was concentrated in vacuo, the crude product was purified by column chromatography with petrol ether-ethyl acetate as an eluent.

1-Methyl-3-phenylquinoline-2(1H)-thione (11a): According to Procedure 4 , a solution of $0.331 \mathrm{~g}(1.00 \mathrm{mmol})$ of 1 -methyl-3phenylquinolinium methylsulfate, $0.128 \mathrm{~g}(4.00 \mathrm{mmol})$ of sulfur, $0.192 \mathrm{~g}$ $(8.00 \mathrm{mmol})$ of $\mathrm{LiOH}$, potassium tert-pentoxide $(1.2 \mathrm{mmol}, 1.7 \mathrm{M}$ solution in toluene) in $10 \mathrm{~mL}$ of toluene was stirred for $6 \mathrm{~h}$ under reflux temperature to give 1 methyl-3-phenylquinoline-2(1H)-thione 11a. Yield $0.050 \mathrm{~g}, 20 \%$, an orange solid, m.p. $118-119{ }^{\circ} \mathrm{C} .{ }^{1} \mathrm{H}$ NMR $\left(600 \mathrm{MHz}, \mathrm{CDCl}_{3}\right): \delta=7.67-$ $7.65(\mathrm{~m}, 2 \mathrm{H}, 5-\mathrm{H}, 7-\mathrm{H}), 7.63-7.60(\mathrm{~m}, 2 \mathrm{H}, 4-\mathrm{H}, 8-\mathrm{H}), 7.50-7.49\left(\mathrm{~m}, 2 \mathrm{H}, 2^{\prime}-\right.$ $\left.\mathrm{H}, 6^{\prime}-\mathrm{H}\right), 7.44-7.41\left(\mathrm{~m}, 2 \mathrm{H}, 3^{\prime}-\mathrm{H}, 5^{\prime}-\mathrm{H}\right), 7.40-7.35$ (m, 2H, 6-H, 4'-H), 4.41 (s, 3H, $\left.\mathrm{NCH}_{3}\right)$ ppm. ${ }^{13} \mathrm{C} \mathrm{NMR}\left(150 \mathrm{MHz}, \mathrm{CDCl}_{3}\right): \delta=185.0$ (o, C2), 143.0 (o, C3), 141.3 (o, C8a), 140.9 (o, C1'), $132.1(+, \mathrm{C} 4), 131.2(+, \mathrm{C} 7), 129.6$ (+, C2', C6'), $129.1(+, \mathrm{C} 5), 128.0\left(+, \mathrm{C} 3^{\prime}, \mathrm{C}^{\prime}\right), 127.9\left(+, \mathrm{C} 4^{\prime}\right), 124.3(+$ C6), 123.8 (o, C4a), $115.8(+, \mathrm{C} 8), 39.1\left(+, \mathrm{NCH}_{3}\right) \mathrm{ppm}$. IR (ATR): 1612, $1595,1563,1493,1443,1414,1372,1346,1324,1299,1233,1215,1180$ 1146, 1121, 1091, 1054, 1028, 1000, 973, 964, 947, 911, 876, 852, 782, $757,746,740,692,646,618,595,576,551,500,481,466,452,447 \mathrm{~cm}$ 1. MS (ESI): $m / z=252.1[\mathrm{M}+\mathrm{H}]^{+}$. HRMS $(\mathrm{ESI}): \mathrm{m} / z$ calcd for $\mathrm{C}_{16} \mathrm{H}_{14} \mathrm{NS}$ $[\mathrm{M}+\mathrm{H}]^{+} 252.0847$, found 252.0848 .

1-Methyl-3-(naphthalen-1-yl)quinoline-2(1H)-thione (11b): According to Procedure 4, a solution of $0.381 \mathrm{~g}(1.00 \mathrm{mmol})$ of 1 -methyl-3(naphthalen-1-yl)quinolinium methylsulfate, $0.128 \mathrm{~g}(4.00 \mathrm{mmol})$ of sulfur, $0.192 \mathrm{~g}(8.00 \mathrm{mmol})$ of $\mathrm{LiOH}$, potassium tert-pentoxide $(1.2 \mathrm{mmol}, 1.7 \mathrm{M}$ solution in toluene) in $10 \mathrm{~mL}$ of toluene was stirred for $6 \mathrm{~h}$ under reflux temperature to give 1 methyl-3-(naphthalen-1-yl)quinoline-2 $(1 \mathrm{H})$-thione 11b. Yield $0.115 \mathrm{~g}, 38 \%$, an orange solid, m.p. $216-218^{\circ} \mathrm{C}$. ${ }^{1} \mathrm{H}$ NMR $(600$ $\left.\mathrm{MHz}, \mathrm{CDCl}_{3}\right): \delta=7.90\left(\mathrm{~d}, J=4.6 \mathrm{~Hz}, 1 \mathrm{H}, 4^{\prime}-\mathrm{H}\right), 7.89\left(\mathrm{~d}, J=4.6 \mathrm{~Hz}, 1 \mathrm{H}, 5^{\prime}-\right.$ H), $7.73(\mathrm{~s}, 1 \mathrm{H}, 4-\mathrm{H}), 7.71-7.65(\mathrm{~m}, 3 \mathrm{H}, 5-\mathrm{H}, 7-\mathrm{H}, 8-\mathrm{H}), 7.62(\mathrm{~d}, J=8.4 \mathrm{~Hz}$, $\left.1 \mathrm{H}, 8^{\prime}-\mathrm{H}\right), 7.55\left(\mathrm{~m}, 1 \mathrm{H}, 3^{\prime}-\mathrm{H}\right), 7.46\left(\mathrm{~m}, 1 \mathrm{H}, 6^{\prime}-\mathrm{H}\right), 7.42-7.36\left(\mathrm{~m}, 3 \mathrm{H}, 6-\mathrm{H}, 2^{\prime}-\right.$ $\left.\mathrm{H}, 7^{\prime}-\mathrm{H}\right), 4.45\left(\mathrm{~s}, 3 \mathrm{H}, \mathrm{NCH}_{3}\right)$ ppm. ${ }^{13} \mathrm{C}$ NMR $\left(150 \mathrm{MHz}, \mathrm{CDCl}_{3}\right): \delta=185.4$ (o, C2), 142.2 (o, C3), 141.2 (o, C8a), 139.0 (o, C1'), 133.7 (o, C4a), 133.4 (+, C4), 131.9 (o, C8a'), $131.4(+$, C7), $129.2(+$, C5), $128.6(+$, C4'), 128.4 $\left(+, C^{\prime}\right), 126.8\left(+, C^{\prime}\right), 126.2(+, C 7), 126.0(+, C 8), 125.9\left(+, 6^{\prime}\right), 125.6$ $\left(+, \mathrm{C}^{\prime}\right), 124.3(+, \mathrm{C} 6), 123.7(\mathrm{o}, \mathrm{C} 4 \mathrm{a}), 115.9(+, \mathrm{C} 8), 38.9\left(+, \mathrm{NCH}_{3}\right) \mathrm{ppm}$. IR (ATR): 1615, 1602, 1594, 1563, 1505, 1494, 1464, 1446, 1410, 1376, $1347,1338,1322,1291,1239,1222,1176,1146,1096,1086,1054,1034$, $1022,1008,970,954,943,904,879,851,796,773,761,747,731,711$, $664,649,637,594,581,563,511,491,474,461,452,447,425,413 \mathrm{~cm}$ 1. MS (ESI): $m / z=302.1[\mathrm{M}+\mathrm{H}]^{+}$. HRMS $(\mathrm{ESI}): \mathrm{m} / z$ calcd for $\mathrm{C}_{20} \mathrm{H}_{16} \mathrm{NS}$ $[\mathrm{M}+\mathrm{H}]^{+}$302.1003, found 302.1003 .

1-Methyl-3-(phenanthren-9-yl)quinoline-2(1H)-thione (11c): According to Procedure 4, a solution of $0.431 \mathrm{~g}(1.00 \mathrm{mmol})$ of 1 -methyl-3(phenanthren-9-yl)quinolinium methylsulfate, $0.128 \mathrm{~g}(4.00 \mathrm{mmol})$ of sulfur, $0.192 \mathrm{~g}(8.00 \mathrm{mmol})$ of $\mathrm{LiOH}$, potassium tert-pentoxide $(1.2 \mathrm{mmol}, 1.7 \mathrm{M}$ solution in toluene) in $10 \mathrm{~mL}$ of toluene was stirred for $6 \mathrm{~h}$ under reflux temperature to give 1 methyl-3-(phenanthren-9-yl)quinoline-2 $(1 \mathrm{H})$-thione 11c. Yield $0.316 \mathrm{~g}, 90 \%$, an orange solid, m.p. $227-228^{\circ} \mathrm{C} .{ }^{1} \mathrm{H}$ NMR $(600$ $\left.\mathrm{MHz}_{\mathrm{CDCl}}\right): \delta=8.75\left(\mathrm{~d}, J=8.3 \mathrm{~Hz}, 1 \mathrm{H}, 5^{\prime}-\mathrm{H}\right), 8.73\left(\mathrm{~d}, J=8.3 \mathrm{~Hz}, 1 \mathrm{H}, 4^{\prime}-\right.$ H), $7.88\left(\mathrm{~d}, J=7.9 \mathrm{~Hz}, 1 \mathrm{H}, 1^{\prime}-\mathrm{H}\right), 7.81(\mathrm{~s}, 1 \mathrm{H}, 4-\mathrm{H}), 7.74-7.66(\mathrm{~m}, 6 \mathrm{H}, 4-\mathrm{H}$, $7-\mathrm{H}, 8-\mathrm{H}, 3^{\prime}-\mathrm{H}, 8^{\prime}-\mathrm{H}, 10^{\prime}-\mathrm{H}$ ), 7.63 (ddd, J=1.2, 7.1, 8.2 Hz, 1H, 6'-H), 7.60 (ddd, $J=0.8,7.1,7.7 \mathrm{~Hz}, 1 \mathrm{H}, 2^{\prime}-\mathrm{H}$ ), 7.49 (ddd, $J=1.1,7.0,8.1 \mathrm{~Hz}, 1 \mathrm{H}, 7^{\prime}-$ $\mathrm{H}$ ), 7.40 (ddd, $J=1.2,7.0,8.0 \mathrm{~Hz}, 1 \mathrm{H}, 6-\mathrm{H}$ ), 4.47 (s, 3H, $\left.\mathrm{NCH}_{3}\right)$ ppm. ${ }^{13} \mathrm{C}$ $\operatorname{NMR}\left(150 \mathrm{MHz}, \mathrm{CDCl}_{3}\right): \delta=185.3$ (o, C2), 142.5 (o, C3), 141.2 (o, C8a), 137.9 (o, C9'), 133.6 (+, C4), 131.9 (o, C10a), $131.4(+, \mathrm{C} 7), 131.1$ (o, C8a'), 130.6 (o, C4a'), 130.5 (o, C4b'), 129.2 (+, C5), 128.9 (+, C1'), 127.3 $\left(+, \mathrm{C}^{\prime} 0^{\prime}\right), 127.0\left(+, \mathrm{C}^{\prime}\right), 126.9\left(+, \mathrm{C}^{\prime}\right), 126.8\left(+, \mathrm{C} 2^{\prime}\right), 126.6\left(+, \mathrm{C} 7^{\prime}\right), 126.6$ (+, C6'), $124.3(+, \mathrm{C} 6), 123.7$ (o, C4a), 123.1 (+, C5), $122.8\left(+, \mathrm{C} 4{ }^{\prime}\right), 115.9$ $(+, \mathrm{C} 8), 38.8\left(+, \mathrm{NCH}_{3}\right)$ ppm. IR (ATR): 1611, 1595, 1563, 1525, 1494 1456, 1449, 1424, 1386, 1369, 1347, 1328, 1309, 1271, 1244, 1233, 1210 , $1185,1166,1144,1110,1097,1088,1038,1009,951,945,915,909,892$ 
$874,857,846,783,759,749,739,721,701,658,636,617,611,582,557$, $529,506,499,486,428 \mathrm{~cm}^{-1}$. MS (ESI): $\mathrm{m} / z=352.1[\mathrm{M}+\mathrm{H}]^{+}$. HRMS (ESI): $\mathrm{m} / \mathrm{z}$ calcd for $\mathrm{C}_{24} \mathrm{H}_{18} \mathrm{NS}[\mathrm{M}+\mathrm{H}]+352.1160$, found 352.1155 .

\section{1-Methyl-3-(4',5',6'-triphenyl-[1,1':2',1'--terphenyl]-3'-yl)quinoline-} 2(1H)-thione (11g): According to Procedure 4, a solution of $0.154 \mathrm{~g}(0.22$ $\mathrm{mmol}$ of 2,3,4,5,6-phenyl-1-(1-methylquinolinium-3-yl)benzene methylsulfate, $0.055 \mathrm{~g}(1.72 \mathrm{mmol})$ of sulfur, $0.042 \mathrm{~g}(1.82 \mathrm{mmol})$ of $\mathrm{LiOH}$, potassium tert-pentoxide ( $0.26 \mathrm{mmol}, 1.7 \mathrm{M}$ solution in toluene) in $5 \mathrm{~mL}$ of toluene was stirred for $6 \mathrm{~h}$ under reflux temperature to give thione $\mathbf{1 1 g}$ Yield $0.050 \mathrm{~g}, 38 \%$, a yellow solid, m.p. $>330{ }^{\circ} \mathrm{C}$. ${ }^{1} \mathrm{H}$ NMR $(600 \mathrm{MHz}$, $\mathrm{CDCl}_{3}$ ): $\delta=7.46$ (ddd, $\left.J=1.6,7.1,8.7 \mathrm{~Hz}, 1 \mathrm{H}, 7-\mathrm{H}\right), 7.41(\mathrm{~s}, 1 \mathrm{H}, 4-\mathrm{H})$, 7.39 (dd, $J=1.5,7.8 \mathrm{~Hz}, 1 \mathrm{H}, 5-\mathrm{H}), 7.37-7.36(\mathrm{~m}, 2 \mathrm{H}, \mathrm{Ph}), 7.30$ (d, $J=8.6$ $\mathrm{Hz}, 1 \mathrm{H}, 8-\mathrm{H}), 7.17$ (ddd, $J=0.7,7.2,8.1 \mathrm{~Hz}, 1 \mathrm{H}, 6-\mathrm{H}), 6.93-6.76(\mathrm{~m}, 23 \mathrm{H}$ $\mathrm{Ph}), 4.00$ (s, 3H, $\mathrm{NCH}_{3}$ ) ppm. ${ }^{13} \mathrm{C}$ NMR (150 MHz, $\left.\mathrm{CDCl}_{3}\right): \delta=184.7$ (o, C2), 142.4 (o, C3), 140.8 (o), 140.65 (o), 140.61 (o), 140.5 (o), 140.03 (o), 139.99 (o, C8a), 138.8 (o), $135.3(+, \mathrm{C} 4), 131.9(+), 131.8(+), 131.6(+)$ $131.4(+), 131.1(+), 130.9(+), 130.5(+$, C7), $128.6(+$, C5), $126.80(+)$ $126.76(+), 126.54(+), 126.47(+), 126.0(+), 125.7(+), 125.2(+), 123.5$ (+, C6), 122.9 (o, C4a), $115.4(+, \mathrm{C} 8), 38.4\left(+, \mathrm{NCH}_{3}\right)$ ppm. IR (ATR): 3055, $3022,2922,2850,1733,1616,1568,1495,1441,1372,1315,1235,1184$ $1148,1095,1072,1043,1027,909,792,740,695,644,552,502,481 \mathrm{~cm}$ 1. HRMS $(\mathrm{ESI}): \mathrm{m} / \mathrm{z}$ calcd for $\mathrm{C}_{46} \mathrm{H}_{33} \mathrm{NNaS}[\mathrm{M}+\mathrm{Na}]^{+} 654.2220$, found 654.2200 .

General procedure for the preparation of selenones (Procedure 5): Under a nitrogen atmosphere, the corresponding salt $(1.00 \mathrm{mmol})$, selenium $(4.00 \mathrm{mmol})$ and $\mathrm{LiOH}(8.00 \mathrm{mmol})$ were suspended in absolute toluene $(10 \mathrm{~mL})$, then potassium tert-pentoxide $(1.20 \mathrm{mmol}, 1.7 \mathrm{M}$ solution in toluene) was added slowly with stirring. The resulting mixture was stirred for $6 \mathrm{~h}$ under reflux at $120^{\circ} \mathrm{C}$. Afterward, the mixture was concentrated in vacuo and the crude product was purified by column chromatography with petrol ether-ethyl acetate as eluent.

1-Methyl-3-phenylquinoline-2(1H)-selenone (12a): According to Procedure 5 , a solution of $0.331 \mathrm{~g}(1.00 \mathrm{mmol})$ of 1 -methyl-3phenylquinolinium methylsulfate, $0.316 \mathrm{~g}$ (4.00 mmol) of selenium, 0.192 $\mathrm{g}(8.00 \mathrm{mmol})$ of $\mathrm{LiOH}$, potassium tert-pentoxide $(1.2 \mathrm{mmol}, 1.7 \mathrm{M}$ solution in toluene) in $10 \mathrm{~mL}$ of toluene was stirred for $6 \mathrm{~h}$ under reflux temperature to give 1 methyl-3-phenylquinoline-2(1H)-selenone 12a. Yield $0.059 \mathrm{~g}$, $20 \%$, a red oil. ${ }^{1} \mathrm{H}$ NMR $\left(600 \mathrm{MHz} \mathrm{CDCl}_{3}\right): \delta=7.74-7.69(\mathrm{~m}, 4 \mathrm{H}, 4-\mathrm{H}, 5$ $\mathrm{H}, 7-\mathrm{H}, 8-\mathrm{H}), 7.47-7.37$ (m, 6H, 6-H, 2'-H, 3'-H, 4'-H, 5'-H, 6'-H), 4.56 (s, $\left.3 \mathrm{H}, \mathrm{NCH}_{3}\right)$ ppm. ${ }^{13} \mathrm{C} \mathrm{NMR}\left(150 \mathrm{MHz}, \mathrm{CDCl}_{3}\right): \delta=186.4(\mathrm{o}, \mathrm{C} 2), 146.7$ (o, C3), 142.7 (o, C1'), 141.5 (o, C8a), $131.4(+, \mathrm{C} 7), 130.4(+, \mathrm{C} 4), 129.7(+$, C2', C6'), 129.3 (+, C5), 127.9 (+, C3', C4', C5'), 125.0 (+, C6), 125.0 (o, C4a), $116.4(+, \mathrm{C} 8), 43.9\left(+, \mathrm{NCH}_{3}\right)$ ppm. ${ }^{77} \mathrm{Se} \mathrm{NMR}\left(114 \mathrm{MHz}, \mathrm{CDCl}_{3}\right) \delta$ $=706.6$ ppm. IR (ATR): 1639, 1591, 1559, 1493, 1443, 1371, 1321, 1234, 1142, 1070, 907, 790, 743, 694, 638, 590, 576, $480 \mathrm{~cm}^{-1}$. HRMS (APCI): $\mathrm{m} / z$ calcd for $\mathrm{C}_{16} \mathrm{H}_{14} \mathrm{NSe}[\mathrm{M}+\mathrm{H}]^{+} 300.0286$, found 300.0275 .

\section{1-Methyl-3-(naphthalen-1-yl)quinoline-2(1H)-selenone}

(12b): According to Procedure 5 , a solution of $0.381 \mathrm{~g}(1.00 \mathrm{mmol})$ of 1 -methyl3-(naphthalen-1-yl)quinolinium methylsulfate, $0.316 \mathrm{~g}(4.00 \mathrm{mmol})$ of selenium, $0.192 \mathrm{~g}(8.00 \mathrm{mmol})$ of $\mathrm{LiOH}$, potassium tert-pentoxide (1.2 mmol, $1.7 \mathrm{M}$ solution in toluene) in $10 \mathrm{~mL}$ of toluene was stirred for $6 \mathrm{~h}$ under reflux temperature to give 1 methyl-3-(naphthalen-1-yl)quinoline2(1H)-selenone 12b. Yield $0.220 \mathrm{~g}, 63 \%$, a red solid, m.p. $196-198{ }^{\circ} \mathrm{C}$ (decomp.). ${ }^{1} \mathrm{H}$ NMR (600 MHz, $\left.\mathrm{CDCl}_{3}\right): 7.90$ (m, 2H, 4'-H, 5'-H), 7.82 (s, $1 \mathrm{H}, 4-\mathrm{H}), 7.80(\mathrm{~d}, J=8.7 \mathrm{~Hz}, 1 \mathrm{H}, 8-\mathrm{H}), 7.75$ (ddd, $J=1.5,7.1,8.7 \mathrm{~Hz}, 1 \mathrm{H}$, $7-\mathrm{H}), 7.70$ (dd, $J=1.5,7.8 \mathrm{~Hz}, 1 \mathrm{H}, 5-\mathrm{H}) 7.61\left(\mathrm{~d}, J=8.4 \mathrm{~Hz}, 1 \mathrm{H}, 8^{\prime}-\mathrm{H}\right), 7.56$ (dd, $\left.J=6.9,8.2 \mathrm{~Hz}, 1 \mathrm{H}, 3^{\prime}-\mathrm{H}\right), 7.47-4.43\left(\mathrm{~m}, 2 \mathrm{H}, 6-\mathrm{H}, 6^{\prime}-\mathrm{H}\right) 7.42$ (dd, $J=$ $1.1,7.0 \mathrm{~Hz}, 1 \mathrm{H}, 2^{\prime}-\mathrm{H}$ ), 7.38 (ddd, $\left.J=1.4,6.9,8.4 \mathrm{~Hz}, 1 \mathrm{H}, 7^{\prime}-\mathrm{H}\right), 4.59$ (s, $\left.3 \mathrm{H}, \mathrm{NCH}_{3}\right) \mathrm{ppm} .{ }^{13} \mathrm{C} \mathrm{NMR}\left(150 \mathrm{MHz}, \mathrm{CDCl}_{3}\right): \delta=186.8$ (o, C2), 145.9 (o, C3), 141.8 (o, 8a), 140.3 (o, C1'), 133.6 (o, C4a'), 132.0 (o, C8a'), 131.8 (+,
C4), $131.7(+$, C7), $129.4(+$, C5), $128.5(+$, C5 '), $128.3(+$, C4 '), $127.0(+$ $\left.\mathrm{C}^{\prime}\right), 126.1\left(+, \mathrm{C}^{\prime}\right), 126.0\left(+, \mathrm{C} 8^{\prime}\right), 125.9(+, \mathrm{C} 6), 125.6\left(+, \mathrm{C} 3^{\prime}\right), 125.0(+$ C6'), 124.9 (o, C4a), $116.4(+, \mathrm{C} 8), 43.7\left(+, \mathrm{NCH}_{3}\right)$ ppm. ${ }^{77} \mathrm{Se} \mathrm{NMR}(114$ $\left.\mathrm{MHz} \mathrm{CDCl}_{3}\right) \delta=716.8$ ppm. IR (ATR): 3051, 3017, 2919, 2849, 2185 1967, 1733, 1610, 1560, 1373, 1238, 1145, 1096, 1067, 795, 773, 761, 581, 491, $423 \mathrm{~cm}^{-1}$. HRMS (APCl): $\mathrm{m} / \mathrm{z}$ calcd for $\mathrm{C}_{20} \mathrm{H}_{16} \mathrm{NSe}[\mathrm{M}+\mathrm{H}]^{+}$ 350.0443 , found 350.0441 .

\section{1-Methyl-3-(phenanthren-9-yl)quinoline-2(1H)-selenone}

(12c): According to Procedure 5, a solution of $0.431 \mathrm{~g}(1.00 \mathrm{mmol})$ of 1 -methyl3-(phenanthren-9-yl)quinolinium methylsulfate, $0.316 \mathrm{~g}(4.00 \mathrm{mmol})$ of selenium, $0.192 \mathrm{~g}(8.00 \mathrm{mmol})$ of $\mathrm{LiOH}$, potassium tert-pentoxide (1.2 mmol, $1.7 \mathrm{M}$ solution in toluene) in $10 \mathrm{~mL}$ of toluene was stirred for $6 \mathrm{~h}$ under reflux temperature to give 1 methyl-3-(phenanthren-9-yl)quinoline$2(1 \mathrm{H})$-selenone $12 \mathrm{c}$. Yield $0.080 \mathrm{~g}, 20 \%$, a red solid, m.p. $271-272{ }^{\circ} \mathrm{C} .{ }^{1} \mathrm{H}$ $\operatorname{NMR}\left(600 \mathrm{MHz}, \mathrm{CDCl}_{3}\right): \delta=8.75\left(\mathrm{~d}, J=8.3 \mathrm{~Hz}, 1 \mathrm{H}, 5^{\prime}-\mathrm{H}\right), 8.73$ (d, $J=8.0$ $\left.\mathrm{Hz}, 1 \mathrm{H}, 4^{\prime}-\mathrm{H}\right), 7.90(\mathrm{~s}, 1 \mathrm{H}, 4-\mathrm{H}), 7.87\left(\mathrm{~d}, J=7.8 \mathrm{~Hz}, 1 \mathrm{H}, 1^{\prime}-\mathrm{H}\right), 7.83(\mathrm{~d}, J=$ $8.6 \mathrm{~Hz}, 1 \mathrm{H}, 8-\mathrm{H}$ ), 7.77 (ddd, $J=1.6,7.1,8.8 \mathrm{~Hz}, 1 \mathrm{H}, 7-\mathrm{H}$ ), 7.72 (dd, $J=$ $1.5,7.8 \mathrm{~Hz}, 1 \mathrm{H}, 5-\mathrm{H}), 7.69-7.65\left(\mathrm{~m}, 3 \mathrm{H}, 3^{\prime}-\mathrm{H}, 8^{\prime}-\mathrm{H}, 10^{\prime}-\mathrm{H}\right), 7.63$ (ddd, $J=$ $1.3,6.9,8.3 \mathrm{~Hz}, 1 \mathrm{H}, 6^{\prime}-\mathrm{H}$ ), 7.60 (ddd, $J=1.0,7.0,7.9 \mathrm{~Hz}, 1 \mathrm{H}, 2^{\prime}-\mathrm{H}$ ), 7.49$7.45\left(\mathrm{~m}, 2 \mathrm{H}, 6-\mathrm{H}, 7^{\prime}-\mathrm{H}\right), 4.61\left(\mathrm{~s}, 3 \mathrm{H}, \mathrm{NCH}_{3}\right) \mathrm{ppm} .{ }^{13} \mathrm{C} \mathrm{NMR}(150 \mathrm{MHz}$ $\mathrm{CDCl}_{3}$ (deptq)): $\delta=186.8(-, \mathrm{C} 2), 146.2(-, \mathrm{C} 3), 141.9(-, \mathrm{C} 8 \mathrm{a}), 139.0(-$ C9'), 132.1 (+, C4), 131.9 (-, C10a'), 131.7 (+, C7), $131.3(-$, C8a'), 130.5 (-, C4a'), 130.5 (-, C4b'), $129.5\left(+\right.$, C5), $129.0\left(+\right.$, C1'), $127.5\left(+\right.$, C9'$\left.^{\prime}\right), 127.1$ $\left(+, 8^{\prime}\right), 126.9\left(+, \mathrm{C}^{\prime}\right), 126.8\left(+, \mathrm{C}^{\prime}\right), 126.6\left(+, \mathrm{C}^{\prime}, \mathrm{C}^{\prime}\right), 125.1(+, \mathrm{C} 6)$, $125.0(-, \mathrm{C} 4 \mathrm{a}), 123.1\left(+, \mathrm{C}^{\prime}\right), 122.9\left(+, \mathrm{C}^{\prime}\right), 116.5(+, \mathrm{C} 8), 43.6\left(+, \mathrm{NCH}_{3}\right)$ ppm. ${ }^{77}$ Se NMR (114 MHz, $\left.\mathrm{CDCl}_{3}\right) \delta=720.4$ ppm. IR (ATR): 1591, 1561, 1449, 1367, 1211, 1135, 1065, 908, 890, 759, 749, 740, 721, 611, 556, $488,428 \mathrm{~cm}^{-1}$. HRMS (ESI): $\mathrm{m} / z$ calcd for $\mathrm{C}_{24} \mathrm{H}_{17} \mathrm{NNaSe}[\mathrm{M}+\mathrm{Na}]^{+} 422.0413$, found 422.0411

\section{1-Methyl-3-(phenanthren-9-yl)-N,N-bis(trimethylsilyl)-1,2-}

dihydroquinolin-2-amine (13): 1-Methyl-3-(phenanthren-9yl)quinolinium hexafluorophosphate $(0.015 \mathrm{~g}, 0.032 \mathrm{mmol})$ and $2 \mathrm{M}$ solution of LiHMDS in THF $(0.02 \mathrm{~mL}, 0.380 \mathrm{mmol})$ were used for the NMR experiment. ${ }^{1} \mathrm{H}$ NMR $\left(600 \mathrm{MHz},\left[\mathrm{D}_{8}\right] \mathrm{THF}\right): \delta=8.76\left(\mathrm{~d}, J=8.3 \mathrm{~Hz}, 1 \mathrm{H}, 5^{\prime}-\right.$ $\mathrm{H}), 8.71\left(\mathrm{~d}, J=8.1 \mathrm{~Hz}, 1 \mathrm{H}, 4^{\prime}-\mathrm{H}\right), 8.18\left(\mathrm{~d}, J=8.1 \mathrm{~Hz}, 1 \mathrm{H}, 1^{\prime}-\mathrm{H}\right), 7.89$ (s, $\left.1 \mathrm{H}, 10^{\prime}-\mathrm{H}\right), 7.85\left(\mathrm{~d}, J=7.9 \mathrm{~Hz}, 1 \mathrm{H}, 8^{\prime}-\mathrm{H}\right), 7.60-7.56\left(\mathrm{~m}, 2 \mathrm{H}, 3^{\prime}-\mathrm{H}, 66^{\prime}-\mathrm{H}\right)$, 7.53-7.49 (m, 2H, 2'-H, 7'-H), 7.17 (t, $J=7.8 \mathrm{~Hz}, 1 \mathrm{H}, 7-\mathrm{H}), 7.13$ (d, $J=7.1$ $\mathrm{Hz}, 1 \mathrm{H}, 5-\mathrm{H}), 6.81(\mathrm{~d}, J=8.1 \mathrm{~Hz}, 1 \mathrm{H}, 8-\mathrm{H}), 6.76(\mathrm{~s}, 1 \mathrm{H}, 4-\mathrm{H}), 6.71(\mathrm{t}, J=$ $7.3 \mathrm{~Hz}, 1 \mathrm{H}, 6-\mathrm{H}), 5.58$ (s, $1 \mathrm{H}, 2-\mathrm{H}), 3.19$ (s, 3H, NCH$), 0.00$ (s, $18 \mathrm{H}$, $\left.\mathrm{NSi}_{2}\left(\mathrm{CH}_{3}\right)_{6}\right)$ ppm. ${ }^{13} \mathrm{C} \mathrm{NMR}\left(150 \mathrm{MHz},\left[\mathrm{D}_{8}\right] \mathrm{THF}\right): \delta=141.5$ (o, C8a), 136.9 (o, C9'), 133.1 (o, C3), 131.8 (o, C10a'), 131.0 (o, C8a'), 130.8 (o, C4a'), 130.0 (o, C4b'), $128.6(+, \mathrm{C} 7), 128.4\left(+, \mathrm{C}^{\prime}\right), 127.4\left(+, \mathrm{C} 10^{\prime}\right), 126.51(+$, $\left.\mathrm{C}^{\prime}\right), 126.46\left(+, \mathrm{C}^{\prime}\right), 126.42(+, \mathrm{C} 4), 126.3\left(+, \mathrm{C}^{\prime}\right), 126.2\left(+, \mathrm{C} 2{ }^{\prime}\right), 126.1$ (+, C3'), 122.9 (+, C5), 122.4 (+, C4'), 121.1 (o, C4a), $116.9(+, \mathrm{C} 6), 111.2$ $(+, \mathrm{C} 8), 83.6(+, \mathrm{C} 2), 35.4\left(+, \mathrm{NCH}_{3}\right), 1.7\left(+, \mathrm{NSi}_{2}\left(\mathrm{CH}_{3}\right)_{6}\right) \mathrm{ppm}$.

\section{Acknowledgments}

Dr. Gerald Dräger, university of Hannover (Germany) is gratefully acknowledged for measuring a part of the HRMS spectra.

Keywords: quinoline $\cdot$ carbene $\cdot$ selenones $\bullet$ heterocycle synthesis $\cdot$ quinolin-2-ylidene

[1] A. Igau, H. Grützmacher, A. Baceiredo, G. Bertrand, J. Am. Chem. Soc 1988, 110, 6463-6466.

[2] A. J. Arduengo, R. L. Harlow, M. Kline, J. Am. Chem. Soc. 1991, 113 361-363. 
[3] a) D. Bourissou, O. Guerret, F. P. Gabbaï, G. Bertrand, Chem. Rev. 2000 100, 39-92; b) F. E. Hahn, M. C. Jahnke, Angew. Chem. Int. Ed. 2008 47, 3122-3172; Angew. Chem. 2008, 120, 3166-3216; c) M. Melaimi, M. Soleilhavoup, G. Bertrand, Angew. Chem. Int. Ed. 2010, 49, 8810-8849 Angew. Chem. 2010, 122, 8992-9032; d) T. Dröge, F. Glorius, Angew. Chem. Int. Ed. 2010, 49, 6940-6952; Angew. Chem. 2010, 122, 7094 7107; e) L. Benhamou, E. Chardon, G. Lavigne, S. Bellemin-Laponnaz, V. César, Chem. Rev. 2011, 111, 2705-2733; f) D. Martin, M. Melaimi, M. Soleihavoup, G. Bertrand, Organometallics 2011, 30, 5304-5313; g) D. J. Nelson, P. Nolan, Chem. Soc. Rev. 2013, 42, 6723-6752; h) M. N Hopkinson, C. Richter, M. Schedler, F. Glorius, Nature 2014, 510, 485496.

[4] a) Z. Guan, K. Hillrichs, C. Ünlü, K. Rissanen, M. Nieger, A. Schmidt, Tetrahedron 2015, 71, $276-282$; b) Z. Guan, J. C. Namyslo, M. H. H. Drafz, M. Nieger, A. Schmidt, Beilstein J. Org. Chem. 2014, 10, 832-840; c) A. G. Tskhovrebov, L. C. E. Naested, E. Solari, R. Scopelliti, K. Severin, Angew.Chem. Int. Ed. 2015, 54,1289-1292; Angew. Chem. 2015, 127, 1305-1308.

[5] a) R. H. Crabtree, J. Organomet. Chem. 2005, 690, 5451-5457. b) F. E. Hahn, M. C. Jahnke, Angew. Chem. Int. Ed. 2008, 47, 3122-3172 Angew. Chem. 2008, 120, 3166-3216; c) A. Schmidt, A. Rahimi, Chem. Commun. 2010, 46, 2995-2997; d) Q. W. Zhao, D. P. Curran, M. Malacria, L. Fensterbank, J. P. Goddard, E. Lacôte, Chem. Eur. J. 2011 17, 9911-9914; e) J. A. Mata, M. Poyatos, Curr. Org. Chem. 2011, 15 3309-3324; f) M. N. Hopkinson, C. Richter, M. Schedler, F. Glorius Nature 2014, 510, 485-496.

[6] a) S. S. Sohn, E. L. Rosen, J. W. Bode, J. Am. Chem. Soc. 2004, 126, 14370-14371; b) E. Enders, O. Niemeier, A. Henseler, Chem. Rev. 2007 107, 5606-5655; c) V. Nair, R. S. Menon, A. T. Biju, C. R. Sinu, R. Rajan Paul, A. Jose, V. Sreekumar, Chem. Soc. Rev. 2011, 40, 5336-5346; d) D. T. Cohen, K. A. Scheidt, Chem. Sci. 2012, 3, 53-57; e) A. Grossmann, D. Enders, Angew. Chem. Int. Ed. 2012, 51, 314-325; Angew. Chem. 2012, 124, 320-332; f) J. Cheng, Z. Huang, Y. R. Chi, Angew. Chem. Int Ed. 2013, 52, 8592-8596; Angew. Chem. 2013, 125, 8754-8758; g) J. Chen, S. Meng, L. Wang, H. Tang, Y. Huang, Chem. Sci. 2015, 6, 4184 4189; h) D. M. Flanigan, F. Romanov-Michailidis, N. A. White, T. Rovis, Chem. Rev. 2015, 115, 9307-9387; i) D. M. Flanigan, T. Rovis, Chem. Sci. 2017, 8, 6566-6569; j) S. Singha, T. Patra, C. G. Daniliuc, F. Glorius, J. Am. Chem. Soc. 2018, 140, 3551-3554.

[7] A. Schmidt, S. Wiechmann, C. F. Otto, Adv. Heterocycl. Chem. 2016, 119, 143-172.

[8] P. Dyson, D. L. Hammick, J. Chem. Soc. 1937, 1724-1725

[9] D. Lavorato, J. K. Terlouw, T. K. Dargel, W. Koch, G. A. McGibbon, H. Schwarz, J. Am. Chem. Soc. 1996, 118, 11898-11904.

[10] S. K. Schneider, P. Roembke, G. R. Julius, H. G. Raubenheimer, W. A. Herrmann, Adv. Synth. Catal. 2006, 348, 1862-1873.

[11] S. K. Schneider, G. R. Julius, C. Loschen, H. G. Raubenheimer, G. Frenking, W. A. Herrmann, Dalton Trans. 2006, 1226-1233.

[12] R. Cordone, H. Taube, J. Am. Chem. Soc. 1987, 109, 8101-8102.

[13] E. Alvarez, S. Conejero, M. Paneque, A. Petronilho, M. L. Poveda, O. Serrano, E. Carmona, J. Am. Chem. Soc. 2006, 128, 13060-13061.
[14] F. Nawaz, K. Mohanan, L. Charles, M. Rajzmann, D. Bonne, O. Chuzel, J. Rodriguez, Y. Coquerel, Chem. Eur. J. 2013, 19, 17578-17583.

[15] R. R. Naredla, B. P. Dash, D. A. Klumpp, Org. Lett. 2013, 15, 4806-4809.

[16] T. K. Dargel, W. Koch, D. J. Lavorato, G. A. McGibbon, J. K. Terlouw, H. Schwarz, Int. J. Mass Spectrom. 1999, 185-187, 925-933.

[17] T. Guo, S. Dechert, S. Meyer, F. Meyer, Organometallics 2012, 31 , 8537-8543.

[18] R. N. Butler, A. M. Gillan, F. A. Lysaght, P. McArdle, D. J. Cunningham, Chem. Soc., Perkin Trans. 11990, 25, 555-564.

[19] R. F. Hudson, Angew. Chem. 1973, 85, 63-84; Angew. Chem. Int. Ed. 1973, 12, 36-56.

[20] a) I. Fleming, Frontier Orbitals and Organic Chemical Reactions, Wiley: London, 1976, p. 66; b) D. Spitzner, Science of Synthesis, 2005, 15, 221 255.

[21] J. A. Cabeza, I. del Río, E. Pérez-Carreño, M. G. Sánchez-Vega, D Vázquez-García, Organometallics 2010, 29, 4464-4471.

[22] a) J. Zhang, E. G. Hübner, J. C. Namyslo, M. Nieger, A. Schmidt, Org Biomol. Chem. 2018, 16, 6801-6808; b) N. L. Ahlburg, O. Doppleb, K. Hillrichs, J. C. Namyslo, E. G. Hübner, A. Schmidt, Heterocycles 2018, 96,1203-1215; c) S. Batsyts, F. J. Ramírez, J. Casado, J. C. Namyslo, A. Schmidt, Z. Naturforsch. 2018, 73, 481-491; d) A.-L. Lücke, S Wiechmann, T. Freese, M. Nieger, T. Földes, I. Pápai, M. Gjikaj, A. Adam, A. Schmidt, Tetrahedron 2018, 74, 2092-2099.

[23] C. Hansch, A. Leo, R. W. Taft, Chem. Rev. 1991, 91, 165-195.

[24] a) A. Schmidt, S. Batsyts, A. Smeyanov, T. Freese, E. G. Hübner, M. Nieger, J. Org. Chem. 2016, 81, 4202-4209.

[25] S. Gómez-Bujedo, M. Alcarazo, C. Pichon, E. Alvarez, R. Fernández, J. M. Lassaletta, Chem. Commun. 2007, 1180-1182.

[26] David R. Lide. CRC Handbook of Chemistry and Physics, 63rd ed.; CRC Press, Boca Raton, 2005.

[27] Lide, D. R., Ed. CRC Handbook of Chemistry and Physics: A readyreference book of chemical and physical data, 75. ed.; CRC Press, Boca Raton, 1994.

[28] a) E. B. Knott, J. Chem. Soc. 1955, 916-927; b) J. Levillain, D. Paquer, A. Sene, M. Vazeux, Synthesis 1998, 1, 99-104; c) A. Angeli, E. Trallori, F. Carta, L. D. C. Mannelli, C. Ghelardini, T. Supuran, ACS Med. Chem. Lett. 2018, 9, 947-951.

[29] a) A. Liske, K. Verlinden, H. Buhl, K. Schaper, C. Ganter Organometallics 2013, 32, 5269-5272; b) K. Verlinden, H. Buhl, W. Frank, C. Ganter, Eur. J. Inorg. Chem. 2015, 2416-2425.

[30] X. Cattoën, D. Bourissou, G. Bertrand, Tetrahedron Lett. 2006, 47, 531534.

[31] G. M. Sheldrick, Acta Crystallogr. 2008, A64, 112-122.

[32] G. M. Sheldrick, Acta Crystallogr. 2015, C71, 3-8

[33] Y. Zhang, M. Wang, P. Li, L. Wang, Org. Lett. 2012, 14, 2206-2209.

[34] S. Smeyanov, Dissertation, Clausthal University of Technology, 2015.

[35] A.-L. Lücke, S. Wiechmann, T. Freese, Z. Guan, A. Schmidt, Z. Naturforsch. B 2016, 71, 643-650.

[36] J.-B. Meng, M.-Q. Shen, X.-H. Wang, C.-H. Kao, R.-J. Wang, H.-G. Wang, T. Matsuura, J. Heterocycl. Chem. 1991, 28, 1481-1484. 


\section{Entry for the Table of Contents}

\section{FULL PAPER}

Series of substituted quinolinium salts were prepared which possess aryl groups or ethynyl groups in position 3 . For $\mathrm{R}=$ aryl $\mathrm{N}$-heterocyclic carbene trapping reactions were performed with sulfur and selenium in the presence of potassium tert-pentoxide and lithium hydroxide.

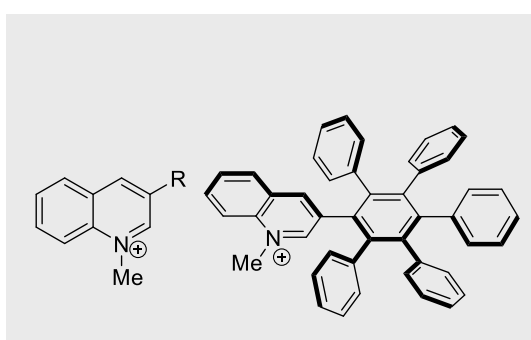

$\mathrm{R}=$ phenyl, naphthalen $-1-y \mathrm{l}$, phenanthren-9-yl, 2-phenylethynyl, 2-(4-Ph-COOMe)ethynyl, 2-(2-Ph-COOMe)ethynyl

\section{N-Heterocyclic Carbenes}

Sviatoslav Batsyts, Roman Vedmid, Jan C. Namyslo, Martin Nieger, and Andreas Schmidt*

Page No. - Page No.

3-Aryl Substituted 1-

Methylquinolinium Salts as Carbene Precursors 